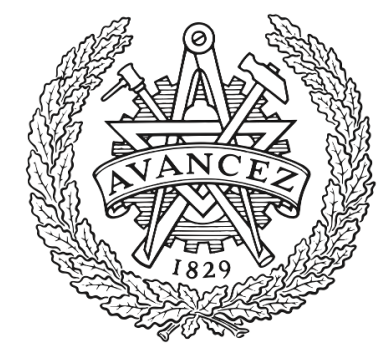

CHALMERS

UNIVERSITY OF TECHNOLOGY

\title{
Dynamics and phonon-induced decoherence of Andreev level qubit
}

Downloaded from: https://research.chalmers.se, 2023-04-26 09:34 UTC

Citation for the original published paper (version of record):

Zazunov, A., Shumeiko, V., Bratus', E. et al (2005). Dynamics and phonon-induced decoherence of Andreev level qubit. Physical Review B - Condensed Matter and Materials Physics, 71.

http://dx.doi.org/10.1103/PhysRevB.71.214505

N.B. When citing this work, cite the original published paper. 


\title{
Dynamics and phonon-induced decoherence of Andreev level qubit
}

\author{
A. Zazunov, V. S. Shumeiko, and G. Wendin \\ Department of Microtechnology and Nanoscience, Chalmers University of Technology, S-41296 Göteborg, Sweden \\ E. N. Bratus' \\ B. Verkin Institute for Low Temperature Physics and Engineering, 61103 Kharkov, Ukraine \\ (Received 27 April 2004; revised manuscript received 16 December 2004; published 2 June 2005)
}

\begin{abstract}
We present a detailed theory for the Andreev level qubit, a system consisting of a highly transmissive quantum point contact embedded in a superconducting loop. The two-level Hamiltonian for Andreev levels interacting with quantum phase fluctuations is derived by using a path integral method. We also derive a kinetic equation describing qubit decoherence due to interaction of the Andreev levels with acoustic phonons. The collision terms are nonlinear due to the fermionic nature of the Andreev states, leading to slow nonexponential relaxation and dephasing of the qubit at temperatures smaller than the qubit level spacing.
\end{abstract}

DOI: 10.1103/PhysRevB.71.214505 PACS number(s): 74.50. + r, 85.25.Dq, 74.25.Kc, 03.67.Lx

\section{INTRODUCTION}

The possibility to employ Andreev bound levels in superconducting contacts for quantum computation has been suggested in Refs. 1 and 2. The proposed Andreev level qubit (ALQ) consists of a highly transmissive, with reflectivity $R$ $\ll 1$, quantum point contact (QPC) embedded in a low inductance superconducting loop. In the ALQ, quantum information is stored in the microscopic two-level system of Andreev bound states in the contact. Hybridization of the clockwise and counterclockwise persistent current states in the ALQ loop is produced by the microscopic processes of electronic back scattering in the QPC. This is different from the macroscopic superconducting flux qubits ${ }^{3-5}$ and charge-phase qubit, ${ }^{6}$ where the hybridization is provided by charge fluctuations on the tunnel junction capacitors. Thus the requirement of large charging energy or large loop inductance is not critical for the ALQ. A single ALQ consists of a pair of Andreev bound levels belonging to the same normal conducting mode in the QPC; a multimode QPC will form a qubit cluster. The way of ALQ operation is similar to the one of the experimentally tested flux qubits ${ }^{3-5}$ - the Andreev levels can be excited by driving a biasing magnetic flux through the qubit loop. ${ }^{7,8,1}$ The read out method is also similar to the flux and charge-phase qubits: the quantum state of the Andreev levels determines the magnitude and direction of the persistent current circulating in the loop, and also the magnitude of the induced flux. Since the quantum information is stored in the microscopic system of Andreev levels, while the access for manipulation and readout is provided by macroscopic persistent currents, the ALQ occupies an intermediate place between the microscopic solid state qubits (such as localized spins on impurities ${ }^{9}$ or quantum dots ${ }^{10}$ ) and macroscopic superconducting qubits.

During the 1990's, the Josephson transport in superconducting QPCs has been intensively investigated, and a number of remarkable experiments has been performed on atomic size metallic QPCs using controllable break junction techniques, ${ }^{11,12}$ as well as on gated quantum constrictions in 2D electron gas confined between superconductors. ${ }^{13}$ The critical Josephson current and current-voltage characteristics have been thoroughly examined in these experiments by applying current or voltage bias. ${ }^{13-16}$ There was, however, one experiment, particularly important in the qubit context, where flux bias was implemented: Koops et al. ${ }^{17}$ inserted a metallic QPC in a SQUID, and evaluated the Josephson current-phase dependence by measuring the induced flux with an inductively coupled SQUID magnetometer. Measurements have only been reported for the equilibrium state. Unfortunately, no experimental attempts to drive the QPC out of equilibrium to some coherent or incoherent excited state have been performed so far.

The purpose of this paper is to present a detailed theory for the Andreev level qubit. ${ }^{2}$ We will also consider the electron-phonon interaction as an "intrinsic" source of qubit decoherence, and derive a kinetic equation for the qubit density matrix. The ALQ Hamiltonian and the kinetic equation are derived by using a path integral method. ${ }^{18-20}$ The central technical difficulty here is to extend the method to contacts with large transparency. This difficulty is overcome by incorporating the exact boundary condition in the QPC action. Another important point discussed in the paper is the role of charge electroneutrality in the junction electrodes, which affects the qubit Hamiltonian.

The fermionic nature of the Andreev levels does not affect the qubit operation and qubit-qubit coupling, but it plays an important role for the qubit decoherence. We find that the electron-phonon collision terms in the kinetic equation for the ALQ differ qualitatively from the Bloch-Redfield master equation $^{21}$ commonly applied to study decoherence of the macroscopic superconducting qubits. ${ }^{22}$ This results in a long phonon-induced decoherence time for the ALQ; at temperatures smaller than the qubit level spacing, both the relaxation and dephasing processes are governed by a power law rather than an exponential law.

The structure of the paper is the following. We discuss the model description of the QPC in Sec. II, and then explain in detail, in Sec. III, the path integral approach for a transmissive QPC: we consider the action for the contact and derive the effective Hamiltonian for Andreev levels interacting with quantum phase fluctuations; the single-particle density matrix and effective current operator are also discussed in this 


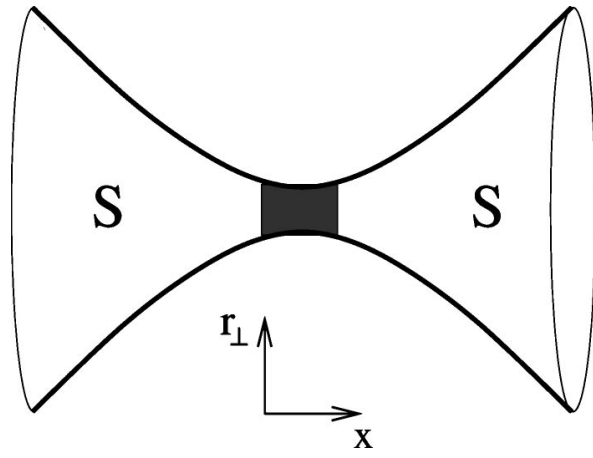

FIG. 1. Adiabatic superconducting constriction with a local scatterer (dark region) in the neck. The length of the constriction is small on the scale of the superconducting coherence length but large on the scale of the Fermi wavelength.

section. In Sec. IV we discuss averaging over fast phase fluctuations and derive an effective Hamiltonian for the qubit. This procedure is extended in Sec. V to the case of two inductively coupled qubits to derive an expression for the direct qubit-qubit interaction. Section VI is devoted to the electron-phonon interaction: we derive an effective action for the Andreev level-phonon interaction and calculate the corresponding collision terms in the equation for the qubit density matrix; we then present solution of the kinetic equation, and evaluate the decoherence rate.

\section{CONTACT HAMILTONIAN}

Let us consider a superconducting quantum point contact with bulk 3D electrodes. We model the contact with a smooth on the Fermi wave length scale (adiabatic) constriction and assume a local scatterer situated in the neck of the constriction (see Fig. 1) producing weak electronic back scattering with reflectivity $R \ll 1$. We further assume that the constriction supports a single conducting mode.

We adopt the mean field approximation for the electrons in the contact described with the Hamiltonian

$$
H_{e}=\int d \mathbf{r} \Psi^{\dagger}(\mathbf{r}, t) h(\mathbf{r}, t) \Psi(\mathbf{r}, t)+\frac{1}{2} C V^{2}(t),
$$

where the first term is the BCS Hamiltonian for the bulk superconducting electrons, $\Psi(\mathbf{r}, t)$ being the two-component Nambu field operator, and the second term describes the charging energy of the contact capacitor $C{ }^{18-20}$ The singleparticle Hamiltonian $h$ in Eq. (1) has the form

$$
\begin{aligned}
h= & {\left[\frac{\left[-i \hbar \nabla-(e / c) \mathbf{A}(\mathbf{r}, t) \sigma_{z}\right]^{2}}{2 m}-\mu+U(\mathbf{r})+e \varphi(\mathbf{r}, t)\right] \sigma_{z} } \\
& +\Delta(\mathbf{r}, t) e^{i \sigma_{z} \chi(\mathbf{r}, t)} \sigma_{x},
\end{aligned}
$$

where $\Delta(\mathbf{r}, t)$ and $\chi(\mathbf{r}, t)$ are, respectively, the modulus and phase of the superconducting order parameter, the potential $U(\mathbf{r})$ accounts for the confinement of electrons within the contact as well as the electron scattering, while $\varphi(\mathbf{r}, t)$ and $\mathbf{A}(\mathbf{r}, t)$ are electromagnetic potentials. The voltage drop at the contact, $V(t)$, in Eq. (1) is related to the discontinuity of the electric potential at the contact, $V(t)=\varphi(-0, t)-\varphi(+0, t)$. To investigate the decoherence effects, we allow the electrons in the electrodes to interact with acoustic phonons, and include the corresponding electron-phonon interaction and phonon terms in the total Hamiltonian of the contact

$$
H_{c}=H_{e}+H_{e-\mathrm{ph}}+H_{\mathrm{ph}} .
$$

It is convenient to introduce the quasiclassical (Andreev) approximation for the superconducting electrons. Following the standard procedure, we eliminate the rapidly space varying potential $U(\mathbf{r})$ by introducing quasiclassical wave functions of the single conducting mode in the left and right electrodes

$$
\Psi(\mathbf{r}, t)=\sum_{\sigma= \pm} \psi_{\perp}\left(\mathbf{r}_{\perp}, x\right) e^{i \sigma \int d x k(x)} \psi^{(\sigma)}(x, t),
$$

and couple these wave functions by means of a normalelectron scattering matrix. In Eq. (4), $\psi^{(\sigma)}(x)$ are slowly varying 1D envelopes for the longitudinal electron motion $(\sigma$ $= \pm$ indicates the direction of the motion); $\psi_{\perp}\left(\mathbf{r}_{\perp}, x\right)$ is a normalized wave function of the transverse motion with en$\operatorname{ergy} E_{\perp}(x) ; p(x)=\hbar k(x)=\sqrt{2 m\left[\mu-E_{\perp}(x)\right]}=m v(x)$ is the quasiclassical longitudinal electronic momentum. The coupling of the quasiclassical envelopes in the left $(L)$ and right $(R)$ electrodes $\psi_{L, R}$ is conveniently described by the transfer matrix $^{7,23}$

$$
\begin{gathered}
\left(\begin{array}{c}
\psi_{L}^{(+)} \\
\psi_{L}^{(-)}
\end{array}\right)(0, t)=\hat{T}\left(\begin{array}{c}
\psi_{R}^{(+)} \\
\psi_{R}^{(-)}
\end{array}\right)(0, t), \\
\hat{T}=\left(\begin{array}{cc}
1 / d & r * / d^{*} \\
r / d & 1 / d^{*}
\end{array}\right) .
\end{gathered}
$$

Here $d$ and $r$ are the energy-independent transmission and reflection amplitudes, respectively. Since any observable quantity is expressed through a bilinear combination of the envelopes with the same $\sigma$, the energy-independent scattering phases can be eliminated from the boundary condition (5); hence, without loss of generality, the scattering amplitudes will be further assumed to be real $d=\sqrt{D}, r=\sqrt{R}$, where $D$ and $R$ are the transmission and reflection coefficients of the contact, respectively.

The electromagnetic potentials $\varphi(\mathbf{r}, t), \mathbf{A}(\mathbf{r}, t)$, and the complex order parameter in Eq. (2) are to be found from the Maxwell equations and the self-consistency equation. It is convenient to present the Hamiltonian in a gauge-invariant form by extracting the phase of the order parameter using a local gauge transformation $\Psi(\mathbf{r}, t) \rightarrow e^{i \sigma_{z} \chi(\mathbf{r}, t) / 2} \Psi(\mathbf{r}, t)$. Then the superfluid momentum $\mathbf{p}_{s}=\hbar \nabla \chi / 2-(e / c) \mathbf{A}$, and the gauge-invariant electric potential $\tilde{\varphi}=\hbar \dot{\chi} / 2+e \varphi$, appear in the quasiclassical Hamiltonian of the electrode

$$
h^{(\sigma)}=\sigma v\left(-i \hbar \partial_{x}\right) \sigma_{z}+\sigma v p_{s}+\tilde{\varphi} \sigma_{z}+\Delta \sigma_{x},
$$

while the phase difference $\phi(t)=\chi_{R}(0, t)-\chi_{L}(0, t)$ appears in the boundary condition 


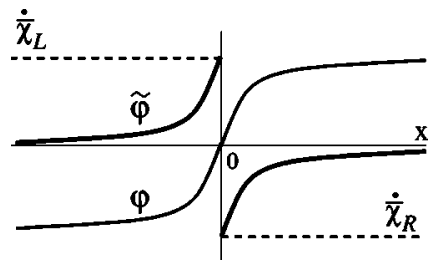

FIG. 2. Spatial distribution of the electric potential $\varphi$ (thin line), gauge invariant potential $\tilde{\varphi}$ (bold line), and the time derivative of the gauge invariant phase $\dot{\bar{\chi}}$ (dashed line) in the vicinity of the contact.

$$
\hat{T} \rightarrow e^{i \sigma_{z} \phi(t) / 2} \hat{T}
$$

In the bulk metallic electrodes with good screening, and at the low frequencies relevant for the problem, the gaugeinvariant fields $\tilde{\varphi}(\mathbf{r}, t), \mathbf{p}_{s}(\mathbf{r}, t)$, are to be found from the electroneutrality condition and current conservation ${ }^{24-26}$

$$
\delta n(\mathbf{r}, t)=0, \quad \nabla \mathbf{j}(\mathbf{r}, t)=0,
$$

where $n(\mathbf{r}, t)$ is the electronic density. In the electrodes, the charge imbalance relaxation yields the equilibrium relation $\widetilde{\varphi}=(\partial n / \partial \mu)^{-1} \delta n$ over distances exceeding the electric field penetration length. Furthermore, in the absence of normal dissipative current, $\mathbf{p}_{s}$ is proportional to the total current density $\mathbf{j}=(\mathrm{en} / \mathrm{m}) \mathbf{p}_{s}$, which is negligibly small far from the contact due to the rapid spreading out of the current in the point contact geometry. Thus the conditions (9) result in complete cancellation of the electromagnetic potentials in the electrodes

$$
\tilde{\varphi}(x, t)=0, \quad p_{s}(x, t)=0 .
$$

Taking into account that the modulus of the order parameter far from the contact is equal to the equilibrium value ${ }^{27} \Delta$ =const, we conclude that the Hamiltonian (7) takes the equilibrium form.

The potential $\tilde{\varphi}$ can be expressed through the electric field $E, \quad \tilde{\varphi}=\hbar \dot{\bar{\chi}} / 2+e \int d x E$, by introducing the gauge-invariant phase $\bar{\chi}=\chi-(2 e / c \hbar) \int d x A=(2 / \hbar) \int d x p_{s}$. The spatial distribution of these quantities is illustrated in Fig. 2. Since $\tilde{\varphi}$ vanishes far from the contact, and $\bar{\chi}$ is constant according to Eq. (10), then the time derivative of the gauge-invariant phase difference across the contact $\dot{\bar{\phi}}=\dot{\bar{\chi}}_{R}(0, t)-\dot{\bar{\chi}}_{L}(0, t)$ is related to the voltage drop $V=\int_{-\infty}^{\infty} d x E$,

$$
\dot{\bar{\phi}}(t)=\frac{2 e V}{\hbar}
$$

(Josephson relation). In the SQUID, this relation is equivalent to the phase versus flux relation $\bar{\phi}=2 e \Phi / \hbar c$ since the voltage drop across the contact is generated by the time variation of the magnetic flux $\Phi$ threading the SQUID. Finally, we notice that the gauge-invariant phase difference $\bar{\phi}$ rather than $\phi$ enters the boundary condition (8), which can be explicitly seen by extracting the Aharonov-Bohm phase from the transfer matrix. Thus the gauge-invariant phase difference remains the only free collective variable whose dynam-

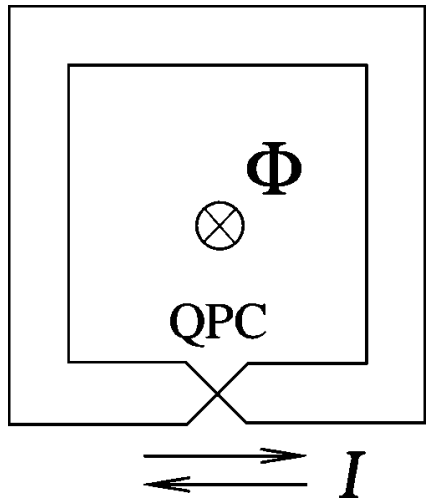

FIG. 3. Sketch of the Andreev level qubit: a low inductance superconducting loop with a quantum point contact (QPC). $\Phi$ is the magnetic flux; the arrows indicate fluctuating persistent currents.

ics is determined by the electrodynamic environment of the contact. Below we will not distinguish between $\bar{\phi}$ and $\phi$, because the difference is negligibly small in the QPC.

Proceeding with a discussion of the interaction of electrons with phonons, we consider only longitudinal acoustic phonons and describe the interaction within the deformation potential approximation

$$
H_{e-\mathrm{ph}}=\gamma \int d \mathbf{r} \nabla \mathbf{u}(\mathbf{r}, t) \Psi^{\dagger}(\mathbf{r}, t) \sigma_{z} \Psi(\mathbf{r}, t),
$$

where $\gamma$ is the deformation potential constant, $\mathbf{u}(\mathbf{r}, t)$ is the phonon field operator,

$$
\mathbf{u}(\mathbf{r})=\sum_{\mathbf{q}} \sqrt{\frac{\hbar}{2 \rho V \Omega_{q}}} \frac{\mathbf{q}}{q}\left(b_{\mathbf{q}} e^{i \mathbf{q} \cdot \mathbf{r}}+b_{\mathbf{q}}^{\dagger} e^{-i \mathbf{q} \cdot \mathbf{r}}\right), \quad \Omega_{q}=s q,
$$

$s$ is the sound velocity, and $\rho$ is the crystal mass density. The Hamiltonian of free phonons has the standard form

$$
H_{\mathrm{ph}}=\sum_{\mathbf{q}} \hbar \Omega_{q}\left(b_{\mathbf{q}}^{\dagger} b_{\mathbf{q}}+1 / 2\right) .
$$

Our strategy will now be to derive an effective Hamiltonian for the Andreev levels including interaction with phonons. If the phase difference would be a classical variable, this derivation can in principle be done by direct truncation of the Hamiltonian (3). However, in the presence of quantum phase fluctuations it is convenient to apply the path integral technique.

\section{CONTACT EFFECTIVE ACTION}

Let us consider the whole system, the QPC and superconducting loop (see Fig. 3), and introduce the path integral representation for the propagator

$$
\mathcal{U}=\int \mathcal{D}^{2} \psi_{L} \mathcal{D}^{2} \psi_{R} \mathcal{D}\left\{X_{\mathbf{q}}\right\} \mathcal{D} \phi e^{i \int d t L_{\mathrm{tot}} / \hbar}, \quad X_{\mathbf{q}}=b_{\mathbf{q}}+b_{\mathbf{q}}^{*} .
$$

The Lagrangian of the system $L_{\text {tot }}$ consists of the contact part $L_{c}$, and the part describing the circulating current in the loop. 
The latter is conveniently combined with the charge term from the electronic Hamiltonian (1) giving the Lagrangian of the loop oscillator $L_{\text {osc }}$

$$
L_{\mathrm{tot}}=L_{c}+L_{\mathrm{osc}}, \quad L_{\mathrm{osc}}=\left(\frac{\hbar}{2 e}\right)^{2}\left(\frac{C}{2}\left(\partial_{t} \phi\right)^{2}-\frac{c^{2}}{2 L}\left(\phi-\phi_{e}\right)^{2}\right),
$$

where $\phi_{e}$ corresponds to the bias magnetic flux and $L$ is the loop inductance. The remaining part of the contact Lagrangian consists, similar to Eq. (3), of the electronic part, the phonon part, and the electron-phonon interaction

$$
L_{c}=L_{e}+L_{\mathrm{ph}}+L_{e-\mathrm{ph}} .
$$

In the quasiclassical approximation, the electronic Lagrangian splits into two parts $L_{\alpha}, \alpha=L, R$, corresponding to the left and right electrodes,

$$
\begin{gathered}
L_{\alpha}=\sum_{\sigma= \pm} \int d x \bar{\psi}_{\alpha}^{(\sigma)}(x, t) \mathcal{L}^{(\sigma)}(x, t) \psi_{\alpha}^{(\sigma)}(x, t), \\
\mathcal{L}^{(\sigma)}(x, t)=i \hbar \partial_{t}+\sigma v i \hbar \partial_{x} \sigma_{z}-\Delta \sigma_{x},
\end{gathered}
$$

and a third part $L_{\mathrm{BC}}$, which accounts for the boundary condition discussed in detail in the next section. Noting that relaxation processes are caused by phonons with small wave vectors compared to the Fermi wave vector $q \ll k$ transitions between the states $\psi^{(+)}$and $\psi^{(-)}$are forbidden, and the electron-phonon Lagrangian can be written in the form

$$
L_{e-\mathrm{ph}}=-\gamma \sum_{\alpha=L, R} \sum_{\sigma= \pm} \int d \mathbf{r}\left|\psi_{\perp}\right|^{2} \bar{\psi}_{\alpha}^{(\sigma)}(x, t) \sigma_{z} \psi_{\alpha}^{(\sigma)}(x, t) \nabla \mathbf{u}(\mathbf{r}, t) .
$$

The phonon Lagrangian is given by

$$
L_{\mathrm{ph}}=\frac{1}{2} \sum_{\mathbf{q}}\left(\hbar / 2 \Omega_{q}\right) X_{\mathbf{q}}\left[\left(i \partial_{t}\right)^{2}-\Omega_{q}^{2}\right] X_{\mathbf{q}} .
$$

\section{A. Boundary condition}

The boundary condition (5) is valid for any contact transparency. To include this boundary condition in the path integral formulation, we introduce an additional term in the Lagrangian ${ }^{2}$

$$
\begin{aligned}
L_{\mathrm{BC}}= & \bar{\eta}(t) \sum_{\sigma}\left[\sqrt{D} e^{-i \sigma_{z} \phi(t) / 4} \psi_{L}^{(\sigma)}(0, t)\right. \\
& \left.-(1+\sqrt{R}) e^{i \sigma_{z} \phi(t) / 4} \psi_{R}^{(\sigma)}(0, t)\right]+ \text { H.c. },
\end{aligned}
$$

where $\eta$ is an auxiliary fermionic Nambu field playing the role of a Lagrange multiplier. Correspondingly, the integration over $\eta$ is to be included in the propagator in Eq. (15), giving the following form for the electronic part of the propagator:

$$
\mathcal{U}_{e}=\int \mathcal{D}^{2} \eta \mathcal{D}^{2} \psi_{L} \mathcal{D}^{2} \psi_{R} e^{i \int d t\left(L_{L}+L_{R}+L_{B C}\right) / \hbar} .
$$

Let us prove that such a Lagrangian indeed generates the boundary conditions (5) and (8). To this end, it is convenient to take a step back and restore a nonquasiclassical form for the fermionic field

$$
\psi(x, t)=\sum_{\sigma} e^{i \sigma \int k d x} \psi^{(\sigma)}(x, t)
$$

in the bulk part of the Lagrangian

$$
\begin{gathered}
L_{L}+L_{R}=\int_{-\infty}^{\infty} d x \bar{\psi}(x, t) \mathcal{L}(x, t) \psi(x, t), \\
\mathcal{L}(x, t)=i \hbar \partial_{t}-\left[\left(-\hbar^{2} / 2 m\right) \partial_{x}^{2}+E_{\perp}-\mu\right] \sigma_{z}-\Delta \sigma_{x} .
\end{gathered}
$$

The dynamic equations and the boundary condition result from the zero variation of the action $S_{e}=\int d t\left(L_{L}+L_{R}+L_{\mathrm{BC}}\right)$ with respect to $\bar{\psi}_{L, R}$ and $\bar{\eta}$,

$$
\frac{\delta S_{e}}{\delta \bar{\psi}_{L, R}(x, t)}=0, \quad \frac{\delta S_{e}}{\delta \bar{\eta}(t)}=0
$$

or, in the explicit form,

$$
\begin{gathered}
\mathcal{L}(x, t) \psi_{L}(x, t)=-\sqrt{D} \delta(x) e^{i \sigma_{z} \phi(t) / 4} \eta(t), \\
\mathcal{L}(x, t) \psi_{R}(x, t)=(1+\sqrt{R}) \delta(x) e^{-i \sigma_{z} \phi(t) / 4} \eta(t),
\end{gathered}
$$

and

$$
\sqrt{D} e^{-i \sigma_{z} \phi(t) / 4} \psi_{L}(0, t)=(1+\sqrt{R}) e^{i \sigma_{z} \phi(t) / 4} \psi_{R}(0, t) .
$$

Integrating Eqs. (26) over $x$ in the close vicinity of $x=0$ yields the relations

$$
\begin{gathered}
\left(\hbar^{2} / 2 m\right) \sigma_{z} \partial_{x} \psi_{L}(0, t)=\sqrt{D} e^{i \sigma_{z} \phi(t) / 4} \eta(t), \\
\left(\hbar^{2} / 2 m\right) \sigma_{z} \partial_{x} \psi_{R}(0, t)=(1+\sqrt{R}) e^{-i \sigma_{z} \phi(t) / 4} \eta(t) .
\end{gathered}
$$

Then introducing again the quasiclassical envelopes and combining Eqs. (27) and (28) with the quasiclassical relation

$$
\partial_{x} \psi_{L, R}(0, t)=i k \sum_{\sigma= \pm} \sigma \psi_{L, R}^{(\sigma)}(0, t)
$$

we get the boundary condition equivalent to Eqs. (5) and (8).

\section{B. Effective action for Andreev levels}

We are now in a position to derive an effective action for the Andreev levels. Following the procedure of Ref. 18, we integrate out fast electronic fields $\psi_{\alpha}$ in Eq. (22),

$$
e^{i S_{\eta}^{0} / \hbar}=\int \prod_{\alpha=L, R} \prod_{\sigma= \pm} \mathcal{D} \bar{\psi}_{\alpha}^{(\sigma)} \mathcal{D} \psi_{\alpha}^{(\sigma)} \exp \left\{\frac{i}{\hbar} \int d t L_{e}\right\} .
$$

We are then left with the effective action $S_{\eta}^{0}$, which contains only variables $\eta(t)$ and $\phi(t)$,

$$
\begin{aligned}
S_{\eta}^{0}= & -\int d t_{1} d t_{2} \bar{\eta}\left(t_{1}\right)\left[D e^{-i \sigma_{z} \phi\left(t_{1}\right) / 4} g\left(t_{1}-t_{2}\right) e^{i \sigma_{z} \phi\left(t_{2}\right) / 4}+(1\right. \\
& \left.+\sqrt{R})^{2} e^{i \sigma_{z} \phi\left(t_{1}\right) / 4} g\left(t_{1}-t_{2}\right) e^{-i \sigma_{z} \phi\left(t_{2}\right) / 4}\right] \eta\left(t_{2}\right),
\end{aligned}
$$

where $g(t)$ is given by the Fourier component 


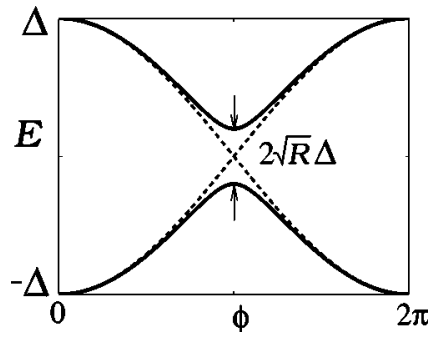

FIG. 4. Spectrum of the Andreev levels in a QPC with finite reflectivity $(R=0.04)$ (solid line). The level anticrossing is produced by electronic backscattering; at $R=0$ the Andreev levels (dashed line) coincide with the current eigenstates.

$$
g_{\omega}=-\frac{\hbar \omega+\Delta \sigma_{x}}{\hbar v \sqrt{\Delta^{2}-(\hbar \omega)^{2}}} .
$$

A connection between the effective action (31) and the Andreev levels can be established by considering the case of time-independent phase $\phi=$ const. Indeed, by writing the effective action in the Fourier representation

$$
\begin{aligned}
S_{\eta}^{0}= & \frac{2(1+\sqrt{R})}{\hbar v} \int \frac{d \omega}{\sqrt{\Delta^{2}-(\hbar \omega)^{2}}} \bar{\eta}_{\omega} \\
& \times\left[\hbar \omega+\Delta\left(\cos \frac{\phi}{2} \sigma_{x}-\sqrt{R} \sin \frac{\phi}{2} \sigma_{y}\right)\right] \eta_{\omega},
\end{aligned}
$$

we identify the spectrum of the system, $\hbar \omega= \pm E_{a}(\phi)$, by calculating the eigenvalues of the matrix inside the brackets

$$
E_{a}(\phi)=\Delta \sqrt{\cos ^{2}(\phi / 2)+R \sin ^{2}(\phi / 2)} ;
$$

this equation coincides with the well known Andreev level spectrum $^{28,29}$ (see Fig. 4). Thus we conclude that the fermionic field $\eta$ represents the Andreev levels.

Proceeding to a time-dependent phase $\phi(t)$, we restrict the rate of time variation to small values $\hbar \partial_{t} \phi / 4 \ll \Delta$. Furthermore, the dynamics of the Andreev levels is also to be slow $E(\phi) \ll \Delta$, which implies that the contact reflectivity must be small $R \ll 1$, the ALQ must be biased at $\phi_{e} \approx \pi$, and the amplitude of the quantum phase fluctuations $\widetilde{\phi}(t)=\phi(t)-\phi_{e}$ must be sufficiently small, $\widetilde{\phi} \ll \phi_{e}$. We emphasize that the constraint on the amplitude of the phase fluctuations is actually provided in our case by the loop geometry of the electrodes having sufficiently small inductance; this constraint is important to suppress the Landau-Zener transitions to the continuum states. Under the imposed conditions, the nonlocal in time kernel $g_{\omega}$, Eq. (32), can be replaced by a constant value (adiabatic approximation) $\sqrt{\Delta^{2}-(\hbar \omega)^{2}} \rightarrow \sqrt{\Delta^{2}-E_{a}^{2}}=\zeta_{e}$ with $E_{a}=E\left(\phi_{e}\right)$, leading to the equation

$$
\begin{aligned}
S_{\eta}^{0}= & \frac{2(1+\sqrt{R})}{\hbar v \zeta_{e}} \int d t \bar{\eta}(t) \\
& \times\left[i \hbar \partial_{t}+\frac{\sqrt{R}}{4} \hbar \dot{\phi} \sigma_{z}+\Delta\left(\cos \frac{\phi}{2} \sigma_{x}-\sqrt{R} \sin \frac{\phi}{2} \sigma_{y}\right)\right] \eta(t) .
\end{aligned}
$$

We then eliminate the term with the phase time derivative in Eq. (35) by transforming $\eta$

$$
\eta \rightarrow\left(\frac{\hbar v \zeta_{e}}{2(1+\sqrt{R})}\right)^{1 / 2} e^{i \sigma_{z} \sqrt{R} \phi / 4} e^{i \sigma_{y} \pi / 4} \eta
$$

and finally arrive at the effective action

$$
S_{\eta}^{0}=\int d t \bar{\eta}(t)\left[i \hbar \partial_{t}-h_{a}\right] \eta(t)
$$

where

$$
h_{a}=\Delta e^{-i \sigma_{x} \sqrt{R} \phi / 2}\left(\cos \frac{\phi}{2} \sigma_{z}+\sqrt{R} \sin \frac{\phi}{2} \sigma_{y}\right)
$$

describes the effective single-particle Hamiltonian for the two-level Andreev system. ${ }^{2}$ The Hamiltonian in Eq. (38) differs by the exponential prefactor from the two-level Hamiltonian derived in Refs. 30 and 31 and further employed in Refs. 32 and 33. This factor appears in the present derivation after electric potential has been included in Eq. (1) to provide the electroneutrality in the electrodes [see text after Eq. (8)]. The Hamiltonians are equivalent under stationary conditions $\partial_{t} \phi=0$ and the difference is not important for the adiabatic dynamics. However, in general, the prefactor is important, e.g., for derivation of the correct equation for the current operator in Eq. (50).

It is instructive to compare the case of the transparent contact considered here with the case of a tunnel contact extensively studied in MQC theory. ${ }^{18-20}$ The physical difference between the two cases is that the Andreev level system in transparent contacts is a slow one, while in tunnel contacts it is fast because the Andreev level energy in tunnel contacts is close to $\Delta$. Within the present formalism, the integration over $\psi$ fields is similar in both cases. However, the next step, the adiabatic approximation in Eqs. (31) and (32) is not allowed in the tunnel limit; instead one should perform also the integration over $\eta$ in Eq. (22), and make an expansion over small $D$. The result of this calculation, presented in Appendix A, coincides with the results of Refs. 18, 19, and 34.

\section{Andreev level density matrix}

Macroscopic properties of the Andreev levels are described by a single-particle density matrix $\rho_{a}(t)$. In particular, the Josephson current through the QPC is defined by this matrix as shown in the next section. The Andreev level density matrix is a $2 \times 2$ matrix in the Nambu space defined via statistical average

$$
\rho_{a}(t)=\left\langle\hat{\eta}(t) \hat{\eta}^{\dagger}(t)\right\rangle,
$$

where $\hat{\eta}$ denotes the fermionic operator corresponding to the Grassmann field $\eta$, and the average is taken over all electronic states. The statistical average in Eq. (39) is represented by a path integral

$$
\rho_{a}(t)=\int \mathcal{D}^{2} \eta \mathcal{D}^{2} \psi_{L, R} \eta(t) \bar{\eta}(t) e^{i S_{e} / \hbar}
$$

or, after the averaging over $\psi_{L, R}$,

$$
\rho_{a}(t)=\int \mathcal{D}^{2} \eta \eta(t) \bar{\eta}(t) e^{i S_{\eta}^{0} / \hbar} .
$$


A dynamic evolution of the Andreev level density matrix is governed by a single-particle Hamiltonian (38). To derive the dynamic equation for $\rho_{a}(t)$ we notice that a free evolution of the Andreev variable, $\eta$, is described by equation

$$
\frac{\delta S_{\eta}^{0}}{\delta \bar{\eta}(t)}=0, \quad i \hbar \partial_{t} \eta=h_{a} \eta
$$

Then calculating the time derivative in Eq. (41), and using Eq. (42) and the corresponding conjugated equation, we obtain

$$
i \hbar \partial_{t} \rho_{a}=\left[h_{a}, \rho_{a}\right] .
$$

Thus the Andreev level dynamics is described by the Liouville equation similar to ordinary quantum mechanical twolevel systems. In equilibrium, the density matrix is diagonal in the Andreev level eigenbasis, and the matrix elements give equilibrium level populations described by the Fermi factors $n_{F}\left( \pm E_{a}\right)$. This is the initial condition for the qubit operation. Since the sum of the level populations $n_{F}\left(E_{a}\right)+n_{F}\left(-E_{a}\right)=1$ is preserved during the time evolution, the density matrix satisfies the normalization condition $\operatorname{Tr} \rho_{a}(t)=1$. For a QPC with reflectivity $R \sim 0.01$, the Andreev level energy $E_{a}(\pi)$ $=\sqrt{R} \Delta$ corresponds to a frequency of the order or larger than $10 \mathrm{GHz}$, which considerably exceeds typical experimental temperatures (below $100 \mathrm{mK}$ ). Thus the QPC should exhibit well pronounced spin $1 / 2$ quantum dynamics, which is the basis for the qubit application.

\section{Andreev level current}

We conclude this section with a derivation of a single particle current operator for the Andreev levels. A common quantum-mechanical expression for the current applied to the fields $\psi_{L, R}$ at $x=\mp 0$ gives

$$
I_{L, R}(t)=\frac{e}{2 m}\left[\bar{\psi}_{L, R}(0, t)\left(-i \hbar \partial_{x}\right) \psi_{L, R}(0, t)+\text { H.c. }\right]
$$

or, using Eq. (28),

$$
I(t)=I_{L, R}(t)=(i e / \hbar) \sqrt{D} \bar{\eta}(t) \sigma_{z} e^{-i \sigma_{z} \phi(t) / 4} \psi_{L}(0, t)+\text { H.c. }
$$

[the current is continuous $I_{L}(t)=I_{R}(t)$ by virtue of Eq. (27)]. The same equation can be obtained by varying the electronic part of the action $S_{e}$ with respect to the phase difference

$$
I(t)=\frac{-2 e}{\hbar} \frac{\delta S_{e}}{\delta \phi(t)} .
$$

It is well known that the Josephson current through a short superconducting constriction is only contributed by the Andreev levels. Having this in mind, we express the Josephson current through the statistically average

$$
\langle I(t)\rangle=\int \mathcal{D}^{2} \eta \mathcal{D}^{2} \psi_{L, R} I(t) e^{i S_{e} / \hbar}
$$

or, equivalently,

$$
\langle I(t)\rangle=2 e i \frac{\delta}{\delta \phi(t)} \int \mathcal{D}^{2} \eta \mathcal{D}^{2} \psi_{L, R} e^{i S_{e} / \hbar} .
$$

Tracing out the fields $\psi_{L, R}$, we get the Josephson current in terms of the Andreev variable

$$
\langle I(t)\rangle=2 e i \frac{\delta}{\delta \phi(t)} \int \mathcal{D}^{2} \eta e^{i S_{\eta}^{0} / \hbar}=\int \mathcal{D}^{2} \eta \bar{\eta}(t) I \eta(t) e^{i S_{\eta}^{0} / \hbar},
$$

where

$$
I=\frac{2 e}{\hbar} \frac{d h_{a}}{d \phi}=-\frac{e}{\hbar} \mathcal{I}(\phi) e^{-i \sigma_{x} \sqrt{R} \phi / 2} \sigma_{z}, \quad \mathcal{I}(\phi)=\Delta D \sin \frac{\phi}{2} .
$$

The $2 \times 2$ matrix $I$ appearing in this equation corresponds to an effective single particle current operator of the Andreev levels. Indeed, comparing equation (41) for the Andreev level density matrix with Eq. (49), we find

$$
\langle I(t)\rangle=\operatorname{Tr}\left(\rho_{a} I\right) .
$$

Apparently the current operator in Eq. (50) does not commute with the Andreev level Hamiltonian (38), $\left[h_{a}, I\right] \neq 0$, which is the consequence of the normal-electron reflection at the QPC. Hence the Andreev level eigenstates consist of superpositions of the current eigenstates, unless $R=0$ (see Fig. 4). Correspondingly, the Andreev level current, defined as an expectation value of the current operator over the Andreev state

$$
I_{a}=\langle I\rangle_{a}= \pm \frac{2 e}{\hbar} \frac{d E_{a}(\phi)}{d \phi}=\mp \frac{e D \Delta^{2}}{2 \hbar E_{a}} \sin \phi,
$$

differs from the current eigenvalues $\mp e \mathcal{I} / \hbar\left[\langle\cdots\rangle_{a}\right.$ denotes here a quantum-mechanical averaging over the Andreev level eigenstate]. Thus the Andreev level current undergoes strong quantum fluctuations. The spectral density of current-current correlation function can be directly calculated by using Eqs. (38) and (50) (cf. Ref. 35)

$$
S(\omega)=\langle I\rangle_{a}^{2} R \tan ^{2}(\phi / 2) \delta\left(\omega-2 E_{a} / \hbar\right) .
$$

\section{AVERAGING OVER PHASE FLUCTUATIONS}

Equation (43) describes the dynamics of the Andreev levels for a given realization of the time dependent phase across the QPC. However, the phase dynamics is strongly coupled to the Andreev levels. The intrinsic dynamics of the phase is governed by the quantum Hamiltonian of the loop oscillator [see Eq. (16)],

$$
H_{\mathrm{osc}}=\frac{p^{2}}{2 M}+\frac{M \omega_{p}^{2} \widetilde{\phi}^{2}}{2}, \quad[p, \phi]=-i \hbar, \quad M=\frac{\hbar^{2}}{8 E_{C}},
$$

where $\omega_{p}=\sqrt{8 E_{L} E_{C} / \hbar^{2}}$ is the plasma frequency of the oscillator determined by the contact charging energy $E_{C}=e^{2} / 2 C$ and the loop inductive energy $E_{L}=(\hbar c / 2 e)^{2}(1 / L)$. Thus the 
whole system is generally a multilevel one. On the other hand, the phase dynamics and the coupling between the loop oscillator and the Andreev levels is a vital part of the ALQ operation: The Andreev levels cannot be manipulated if the phase dynamics were frozen because, first, any manipulation requires variation of the current and secondly, the read out of the Andreev levels can be only performed via measuring the quantum state of the loop oscillator. An obvious way to solve this problem and preserve the qubit property of the coupled system is to "enslave" the loop oscillator by choosing the oscillator level spacing $\hbar \omega_{p}$ much larger than the Andreev level spacing $\hbar \omega_{p} \gg 2 E_{a}$. Then the Andreev state evolution will not excite the oscillator, which will remain in the ground state and adiabatically follow the evolution of the Andreev levels. This implies that the phase is a fast variable which should be averaged out, leading to an effective qubit Hamiltonian.

To facilitate the averaging procedure we take advantage of the small amplitude of the phase fluctuations $\widetilde{\phi}=\phi-\phi_{e}$ $\ll \phi_{e}$, which was already assumed when proceeding from Eq. (33) to Eq. (35). This assumption is justified by the large inductive energy $E_{L} \gg E_{J} \approx \Delta$ and it allows us to expand the Andreev level Hamiltonian (38) in terms of small $\widetilde{\phi}$; then proceeding to the current eigenbasis $\eta \rightarrow e^{-i \sigma_{x} \backslash \bar{R} \phi_{e} / 4} \eta$, we obtain

$$
h_{a}=\Delta\left(\cos \frac{\phi_{e}}{2} \sigma_{z}+\sqrt{R} \sin \frac{\phi_{e}}{2} \sigma_{y}\right)-\frac{\mathcal{I}\left(\phi_{e}\right)}{2} \widetilde{\phi} \sigma_{z}=h_{a}^{0}+h_{\mathrm{int}} .
$$

Averaging over fast phase fluctuations can be done directly in Eq. (15) by performing explicit integration over phase. However, there is a simpler way to get the same result. Equation (43) holds for a fluctuating phase provided the oscillator is not excited. It can be viewed as the diagonal part with respect to $\phi$ of a more general equation for a full density matrix $\rho\left(\sigma \phi, \sigma^{\prime} \phi^{\prime}\right)$ for the Andreev levels plus oscillator system

$$
i \hbar \partial_{t} \rho=\left[h_{a}^{0}+h_{\mathrm{int}}+H_{\mathrm{osc}}, \rho\right] .
$$

The interaction between the Andreev levels and the oscillator given by the second term in Eq. (55) displaces the oscillator steady state from the origin by $\pm \mathcal{I} / 2 M \omega_{p}^{2}$ depending on the direction of the current in the junction or, equivalently, the state of the Andreev levels (see Fig. 5). We eliminate this term in Eq. (55) by applying the transformation

$$
h_{a} \rightarrow e^{i A p} h_{a} e^{-i A p}, \quad A=\frac{\mathcal{I}\left(\phi_{e}\right) \sigma_{z}}{2 M \hbar \omega_{p}^{2}},
$$

and then average the resulting Hamiltonian over the oscillator ground state, taking into account the relation $\left\langle e^{i A} p\right\rangle_{0}$ $=\exp \left(-A^{2}\left\langle p^{2}\right\rangle_{0} / 2\right)\left[\langle\cdots\rangle_{0}\right.$ indicates averaging over the oscillator ground state]. As a result we get an effective Hamiltonian

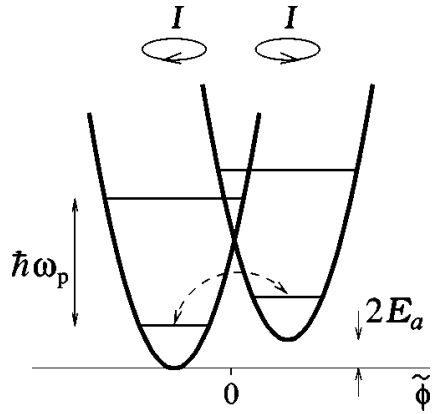

FIG. 5. Potential energy diagram for two displaced oscillator states corresponding to the different current states in the point contact (shown as arrowed circles). The plasma frequency $\omega_{p}$ is large compared to the Andreev level spacing; the oscillator remains in the ground state during the qubit evolution (dashed arrow).

$$
\left\langle h_{a}\right\rangle_{0}=\Delta\left(\cos \frac{\phi_{e}}{2} \sigma_{z}+\sqrt{R^{*}} \sin \frac{\phi_{e}}{2} \sigma_{y}\right)=h_{q}\left(\phi_{e}\right),
$$

where the bare contact reflectivity $R$ is renormalized by the phase fluctuations

$$
R^{*}=e^{-2 \lambda} R, \quad \lambda=\mathcal{I}^{2}\left(\phi_{e}\right) / 4 M \hbar \omega_{p}^{3} .
$$

This renormalization effect can be understood as the effect of inertia of the loop oscillator, which hinders the current variations, i.e., it works against the effect of the electronic back scattering at the contact responsible for the hybridization of the current states (see discussion in the end of the Sec. III D). The renormalization effect becomes increasingly strong in the limit of a classical oscillator with large "mass." Because of renormalization of the contact reflectivity, the Andreev level spectrum is modified,

$$
E_{a}\left(\phi_{e}\right) \rightarrow E_{a}^{*}\left(\phi_{e}\right)=\Delta \sqrt{\cos ^{2}\left(\phi_{e} / 2\right)+R * \sin ^{2}\left(\phi_{e} / 2\right)},
$$

and the qubit frequency reduced. This might be important for practical applications, because it would allow one to reduce the qubit frequency by choosing the circuit parameters rather than by tuning the contact reflectivity.

Equation (58) gives an effective Hamiltonian $h_{q}$ for the ALQ in the absence of interaction with phonons. Similarly, averaged over phase fluctuation, the density matrix $\langle\rho\rangle_{0}$ gives the density matrix for the ALQ. Keeping the same notation $\rho$ for the qubit density matrix, we finally arrive at the equation for the free evolution of the ALQ

$$
i \hbar \partial_{t} \rho=\left[h_{q}\left(\phi_{e}\right), \rho\right] .
$$

This equation is sufficient to describe the ALQ manipulation (by driving the biasing flux) and read out (by measuring the induced current),, 2 and also the qubit-qubit interaction, which is discussed in the next section.

\section{INDUCTIVE QUBIT-QUBIT COUPLING}

Our treatment of the interaction of the Andreev levels with phase fluctuations can be easily extended on the case of 
several inductively coupled SQUIDs to describe direct qubitqubit coupling. Let us consider as an example two SQUIDs with different QPC reflectivities $R_{1} \neq R_{2}$, identical circuit parameters $C$ and $L$, and mutual inductance $\mathcal{M}$. Then the inductance terms in the Lagrangian (16) written for the two qubits will take the form

$$
\frac{1}{2}\left(\frac{\hbar c}{2 e}\right)^{2} \widetilde{\phi}^{T} \hat{L}^{-1} \widetilde{\phi}, \quad \widetilde{\phi}=\left(\begin{array}{l}
\widetilde{\phi}_{1} \\
\widetilde{\phi}_{2}
\end{array}\right), \quad \hat{L}=\left(\begin{array}{cc}
L & \mathcal{M} \\
\mathcal{M} & L
\end{array}\right),
$$

where $\widetilde{\phi}_{1,2}$ are fluctuating phases in the first and second qubits. By introducing the normal modes for the LC oscillators $\widetilde{\phi} \rightarrow e^{-i \tau_{y} \pi / 4} \widetilde{\phi}$ we obtain the two-qubit Hamiltonian on form similar to Eq. (55)

$$
\begin{aligned}
H= & \sum_{i=1,2}\left[\Delta\left(\cos \frac{\phi_{e, i}}{2} \sigma_{z i}+\sqrt{R_{i}} \sin \frac{\phi_{e, i}}{2} \sigma_{y i}\right)\right. \\
& \left.-\frac{\widetilde{\phi}_{i}}{2 \sqrt{2}}\left[(-1)^{i+1} \mathcal{I}_{1} \sigma_{z 1}+\mathcal{I}_{2} \sigma_{z 2}\right]+H_{\mathrm{osc}, i}\right],
\end{aligned}
$$

where $H_{\text {osc, } i}$ describes the normal oscillator with frequency $\omega_{p i}=c / \sqrt{C(L \pm \mathcal{M})}$, and the index $i=1,2$ refers to the first and second qubit. Then we apply to Eq. (63) the similar transformations as in the previous section, namely, we eliminate the terms linear in $\widetilde{\phi}_{i}$ by means of a canonical transformation $H \rightarrow \exp \left(i \sum_{i} A_{i} p_{i}\right) H \exp \left(-i \sum_{i} A_{i} p_{i}\right)$, with $A_{i}$ $=\left[(-1)^{i+1} \mathcal{I}_{1} \sigma_{z 1}+\mathcal{I}_{2} \sigma_{z 2}\right] /\left(2 \sqrt{2} M \hbar \omega_{i}^{2}\right)$, and then average the transformed Hamiltonian over the ground state of the normal oscillators. As a result, we obtain an effective two-qubit Hamiltonian including direct qubit-qubit coupling

$$
H=h_{q 1}+h_{q 2}+(e / \hbar c)^{2} \mathcal{M} \mathcal{I}_{1} \mathcal{I}_{2} \sigma_{z 1} \sigma_{z 2} .
$$

The renormalized contact reflectivities are now given by $R_{i}^{*}$ $=R_{i} \exp \left[-\left(\mathcal{I}_{i}^{2} / 4 \hbar M\right)\left(\omega_{1}^{-3}+\omega_{2}^{-3}\right)\right]$.

It is worth mentioning that the two-qubit configuration may be also realized with a single SQUID containing a QPC with two conducting modes. In this case, we have the two Andreev level Hamiltonians coupled to the same loop oscillator

$$
H=\sum_{i=1,2}\left(h_{a, i}^{0}-\frac{\mathcal{I}_{i}\left(\phi_{e}\right)}{2} \widetilde{\phi} \sigma_{z i}\right)+H_{\mathrm{osc}} .
$$

Averaging over the phase fluctuations, we arrive at the same interaction Hamiltonian as in Eq. (64) but with the loop inductance $L$ substituting for $-\mathcal{M}$. Thus we come to an interesting conclusion that the effect of the phase fluctuations not only reduces the bare contact reflectivity but also introduces effective coupling of the Andreev levels of different conducting modes in a multimode QPC.

\section{ANDREEV LEVEL-PHONON INTERACTION}

\section{A. Effective action}

In this section we take electron-phonon interaction into account. We start with the derivation of an effective action for the Andreev level-phonon interaction. To this end we repeat the calculation of the previous section adding the Lagrangian $L_{e-\mathrm{ph}}$, Eq. (19), to Eq. (30). By retaining in the electrode Green functions only the first-order correction in the small interaction constant $\gamma$, we arrive at the following action:

$$
\begin{aligned}
S_{\eta}= & -\int d t_{1} d t_{2} \bar{\eta}\left(t_{1}\right)\left[D e^{-i \sigma_{z} \phi\left(t_{1}\right) / 4} G_{L}\left(t_{1}, t_{2}\right) e^{i \sigma_{z} \phi\left(t_{2}\right) / 4}\right. \\
& \left.+(1+\sqrt{R})^{2} e^{i \sigma_{z} \phi\left(t_{1}\right) / 4} G_{R}\left(t_{1}, t_{2}\right) e^{-i \sigma_{z} \phi\left(t_{2}\right) / 4}\right] \eta\left(t_{2}\right),
\end{aligned}
$$

where the Green functions $G_{L, R}$ read

$$
\begin{aligned}
G_{\alpha}\left(t_{1}, t_{2}\right)= & \sum_{\sigma= \pm}\left[g_{\alpha}^{(\sigma)}\left(0,0 ; t_{1}-t_{2}\right)+\gamma \int d \mathbf{r}\left|\psi_{\perp}\right|^{2}\right. \\
& \left.\times \int d t g_{\alpha}^{(\sigma)}\left(0, x ; t_{1}-t\right) \nabla \mathbf{u}(\mathbf{r}, t) \sigma_{z} g_{\alpha}^{(\sigma)}\left(x, 0 ; t-t_{2}\right)\right] .
\end{aligned}
$$

In this equation, the quantities $g_{L . R}^{(\sigma)}$ refer to the different parts $g^{(\sigma)}\left(x<0, x^{\prime}<0 ; t\right)$ and $g^{(\sigma)}\left(x>0, x^{\prime}>0 ; t\right)$, respectively, of the translation-invariant free electron Green function $g^{(\sigma)}(x$ $\left.-x^{\prime}, t\right)$, which obeys the equation

$$
\left[i \hbar \partial_{t}+\sigma \sigma_{z} v i \hbar \partial_{x}-\Delta \sigma_{x}\right] g^{(\sigma)}\left(x-x^{\prime}, t\right)=\delta\left(x-x^{\prime}\right) \delta(t) .
$$

The Green functions $g_{\alpha}^{(\sigma)}$ in Eq. (67) are explicitly given by

$$
g_{L, R}^{(\sigma)}(x, 0 ; t)=-\Theta(\mp x) \frac{e^{-|x| \zeta_{t} / \hbar v}}{2 \hbar v \zeta_{t}}\left[i \hbar \partial_{t}+\Delta \sigma_{x} \mp \sigma i \zeta_{t} \sigma_{z}\right] \delta(t),
$$

where $\zeta_{t}$ is given by the Fourier component $\zeta_{\omega}$ $=\sqrt{\Delta^{2}-(\hbar \omega)^{2}}$.

Proceeding to the adiabatic approximation discussed in the previous section $\left(\zeta_{\omega} \rightarrow \zeta_{e}\right)$, and performing the transformation (36), we arrive at the following effective action for the Andreev level-phonon interaction:

$$
S_{\eta-\mathrm{ph}}=-\gamma \int d t \int d \mathbf{r}\left|\psi_{\perp}\right|^{2} n(x, t) \nabla \mathbf{u}(\mathbf{r}, t)
$$

where

$$
n(x, t)=\frac{e^{-2|x| \zeta_{e} / \hbar v}}{4 \hbar v \zeta_{e}}(1+\operatorname{sgn} x \sqrt{R}) \Lambda(x, t)
$$

and

$$
\begin{aligned}
\Lambda(x, t)= & \hbar^{2}\left(\partial_{t} \bar{\eta}\right) \sigma_{x}\left(\partial_{t} \eta\right)+\hbar \Delta\left(\partial_{t} \bar{\eta} e^{-i \sigma_{x}(\sqrt{R}-\operatorname{sgn} x) \phi_{e} / 2} \sigma_{y} \eta+\text { H.c. }\right) \\
& -E_{a}^{2} \bar{\eta} \sigma_{x} \eta .
\end{aligned}
$$

Taking into account the zero-order dynamic equation with respect to $\gamma$, Eq. (42), and putting $\phi=\phi_{e}$

$$
i \hbar \partial_{t} \eta=\Delta e^{-i \sigma_{x} \sqrt{R} \phi_{e} / 2}\left(\cos \frac{\phi_{e}}{2} \sigma_{z}+\sqrt{R} \sin \frac{\phi_{e}}{2} \sigma_{y}\right) \eta,
$$

we obtain for the quantity $n(x, t)$, Eq. (71), the following expression: 


$$
n(x, t)=(\sqrt{R} / 2) \kappa \operatorname{sgn} x e^{-2 \kappa|x|} \bar{\eta} \sigma_{x} \eta,
$$

where $\kappa=\zeta_{e} / \hbar v$. Finally, integrating over $\mathbf{r}$ in the action (70), we obtain the effective interaction on the form

$$
S_{\eta-\mathrm{ph}}=-\int d t \sum_{\mathbf{q}} \gamma_{\mathbf{q}} X_{\mathbf{q}} \bar{\eta} \sigma_{x} \eta,
$$

where $\gamma_{\mathbf{q}}$ is the effective constant of the Andreev levelphonon coupling

$$
\begin{gathered}
\gamma_{\mathbf{q}}=\gamma \kappa \sqrt{\frac{\hbar \Omega_{q} R}{2 \rho V s^{2}}} \int_{0}^{+\infty} d x F\left(\mathbf{q}_{\perp}, x\right) e^{-2 \kappa x} \sin \left(q_{x} x\right), \\
F\left(\mathbf{q}_{\perp}, x\right)=\int d \mathbf{r}_{\perp}\left|\psi_{\perp}(\mathbf{r})\right|^{2} \mathbf{e}^{\mathbf{i} \cdot \mathbf{q}_{\perp} \mathbf{r}_{\perp} .}
\end{gathered}
$$

We notice that the Andreev level-phonon interaction in Eq. (75) has purely transverse origin, i.e., while inducing interlevel transitions and hence relaxation, it does not produce any additional dephasing to the one associated with the relaxation.

It is important to mention that the effective coupling constant is proportional to $\sqrt{R}$, and it turns to zero in the case of perfectly transparent constriction. This results from the already mentioned fact that the relevant phonons have small wave vectors and are not able to provide large momentum transfer $(\sim 2 \hbar k)$ during scattering with electrons.

The effective action in Eqs. (75) and (76) was derived for a given realization of the time dependent phase. To take into account the effect of phase fluctuation, we have to apply the transformation in Eq. (57) to the action (75); the Lagrange form of the transformation reads

$$
\eta \rightarrow \exp \left(i \frac{\mathcal{I}\left(\phi_{e}\right) \sigma_{z}}{2 \hbar} \int^{t} d \tau \widetilde{\phi}(\tau)\right) \eta
$$

Then the integration over the phase adds the factor $e^{-\lambda}$ to the action, see Eq. (59), which simply implies a renormalization of the coupling constant

$$
\gamma_{\mathbf{q}} \rightarrow \gamma_{\mathbf{q}}^{*}=e^{-\lambda} \gamma_{\mathbf{q}}
$$

This result can be expected: since the Andreev level-phonon coupling is transverse in the current basis and depends on the contact reflectivity, the renormalized reflectivity $R^{*}$ rather than the bare one has to enter the coupling constant $\gamma_{q}^{*}$ $=\gamma_{q}\left(R^{*}\right)$; this is consistent with Eqs. (76) and (78).

\section{B. Kinetic equation}

Qubit decoherence is usually described through collision terms in the Liouville equation for the qubit density matrix taking into account the interaction with an environment. Our goal will now be to derive the phonon-induced collision terms in Eq. (61) for the ALQ density matrix, and to evaluate the decoherence of the ALQ.

While the description of free qubit evolution was possible in terms of the single-particle density matrix, evaluation of the collision terms goes beyond the single-particle approximation and requires the knowledge of electronic two-particle correlation functions. This is because the Andreev levels do not form a rigorously isolated system but rather belong to a large fermionic system of the superconducting electrons in the contact electrodes. Thus to derive the collision terms, we apply the many-body Keldysh-Green function technique ${ }^{36}$ combined with the path integral approach. The method described below automatically takes into account many-body effects in the form of the Pauli exclusion principle, leading to a nonlinear form of the collision terms and eventually to the suppression of decoherence.

The starting point of the derivation is Eqs. (15) and (22) for the propagator, in which the integration over the fast fermionic fields $\psi_{L, R}$ and phase $\phi$ has been performed while integration over the phonons and Andreev states remains

$$
\mathcal{U}=\int \mathcal{D}\left\{X_{\mathbf{q}}\right\} \mathcal{D}^{2} \eta e^{i S / \hbar} .
$$

The time evolution in the action now follows along the Keldysh contour ${ }^{37} C_{K}, S=\int_{C_{K}} d t L$, which goes from $-\infty$ to $+\infty$, and then backwards. ${ }^{20,38,39}$ The interaction is supposed to be switched on and off adiabatically at the remote past $t$ $=-\infty$ and the phonon bath is supposed to be in thermal equilibrium. The Lagrangian $L$ has the form

$$
L=\bar{\eta}\left(i \hbar \partial_{t}-E_{a}^{*} \sigma_{z}\right) \eta-\sum_{\mathbf{q}} \gamma_{\mathbf{q}}^{*} X_{\mathbf{q}} \bar{\eta} \sigma_{x} \eta+L_{\mathrm{ph}},
$$

where $L_{\mathrm{ph}}$ is given by Eq. (20). To reduce the time integration along the Keldysh contour to an ordinary time integral, we distinguish forward and backward branches of the contour by labeling them with index $s=1,2$, and introduce the two-component fields $\eta^{s}$ and $X_{\mathbf{q}}^{s}$. Since the action is local in time, it can be rewritten as $S=\int_{-\infty}^{+\infty} d t\left(L^{1}-L^{2}\right)$, where $L^{S}$ $=L\left[\bar{\eta}^{s}, \eta^{s}, X_{\mathbf{q}}^{s}\right]$.

The first step of the derivation is to integrate out the phonon fields, which will give rise to an effective self-interaction for the field $\eta$,

$$
\begin{aligned}
S_{\text {int }}[\bar{\eta}, \eta]= & -\frac{1}{2} \int d t d t^{\prime}\left[\bar{\eta}(t) \sigma_{x} \eta(t)\right]^{s} \\
& \times\left[\tau_{z} \check{D}\left(t-t^{\prime}\right) \tau_{z}\right]^{s s^{\prime}}\left[\bar{\eta}\left(t^{\prime}\right) \sigma_{x} \eta\left(t^{\prime}\right)\right]^{s^{\prime}},
\end{aligned}
$$

with the kernel $\check{D}\left(t-t^{\prime}\right)$ given by

$$
\begin{gathered}
\check{D}\left(t-t^{\prime}\right)=\sum_{\mathbf{q}}\left|\gamma_{\mathbf{q}}^{*}\right|^{2} \check{D}_{\mathbf{q}}\left(t-t^{\prime}\right), \\
D_{\mathbf{q}}^{s s^{\prime}}\left(t-t^{\prime}\right)=-(i / \hbar)\left\langle T_{C}\left(X_{\mathbf{q}}^{s}(t) X_{\mathbf{q}}^{s^{\prime}}\left(t^{\prime}\right)\right)\right\rangle,
\end{gathered}
$$

where $\check{D}_{\mathbf{q}}\left(t-t^{\prime}\right)$ is the equilibrium Keldysh Green function of the phonons represented by the $2 \times 2$ matrix in the Keldysh space. In these equations, $T_{C}$ is the time-ordering operator on the Keldysh contour and $\tau_{z}$ is the Pauli matrix operating in the Keldysh space; summation over repeated indices is implied.

The next step is to take advantage of the weak electronphonon interaction, and to decouple the four-fermionic interaction term in Eq. (81) by introducing the HubbardStratonovich field $\mathcal{G}_{\alpha \beta}^{s s^{\prime}}\left(t, t^{\prime}\right)$, which is a matrix in the 
Keldysh-Nambu-time space. Before doing this, it is convenient to explicitly extract the small parameter $\lambda_{\mathrm{ph}}$, which determines the electron-phonon coupling strength, from the kernel $\check{D}$ in Eq. (81) by redefining the kernel $\check{D} \rightarrow \lambda_{\mathrm{ph}} \check{D}$ $\left[\lambda_{\mathrm{ph}} \sim(v / s)\left(\omega / \omega_{D}\right)^{2} \ll 1, \omega_{D}\right.$ is the Debye frequency]. As a result, we get

$$
\begin{aligned}
\mathcal{U}= & \int \mathcal{D} \check{\mathcal{G}} \mathcal{D}^{2} \eta \exp \left[( i / \hbar ) \int d t d t ^ { \prime } \overline { \eta } ^ { s } ( t ) \left[\mathcal{L}\left(t, t^{\prime}\right) \tau_{z}\right.\right. \\
& \left.\left.-\tau_{z} \check{\Sigma}\left(t, t^{\prime}\right) \tau_{z}\right]^{s s^{\prime}} \eta^{s^{\prime}}\left(t^{\prime}\right)+i W[\check{\mathcal{G}}]\right],
\end{aligned}
$$

where $\mathcal{L}\left(t, t^{\prime}\right)=\left(i \hbar \partial_{t}-E_{a}^{*} \sigma_{z}\right) \delta\left(t-t^{\prime}\right)$ is diagonal in the Keldysh space and

$$
\begin{aligned}
& W[\check{\mathcal{G}}]= \frac{\hbar}{2 \lambda_{\mathrm{ph}}} \int d t d t^{\prime} \mathcal{G}_{\alpha \beta}^{s s^{\prime}}\left(t, t^{\prime}\right) \\
& \times\left[\tau_{z} \check{D}\left(t-t^{\prime}\right) \tau_{z}\right]^{s s^{\prime}}\left[\sigma_{x} \check{\mathcal{G}}\left(t^{\prime}, t\right) \sigma_{x}\right]_{\beta \alpha}^{s^{\prime} s}, \\
& \sum_{\alpha \beta}^{s s^{\prime}}\left(t, t^{\prime}\right)=i \hbar D^{s s^{\prime}}\left(t-t^{\prime}\right)\left[\sigma_{x} \check{\mathcal{G}}\left(t, t^{\prime}\right) \sigma_{x}\right]_{\alpha \beta}^{s s^{\prime}} .
\end{aligned}
$$

Equation (83) describes the dynamics of the field $\eta$ interacting with the effective field $\breve{\mathcal{G}}$. In terms of the Keldysh-Green function for the field $\eta$,

$$
G_{\alpha \beta}^{s s^{\prime}}\left(t, t^{\prime}\right)=-(i / \hbar)\left\langle T_{C}\left(\eta_{\alpha}^{s}(t) \eta_{\beta}^{\dagger s^{\prime}}\left(t^{\prime}\right)\right)\right\rangle,
$$

this evolution is described by the Dyson equation

$$
\left(\mathcal{L} \tau_{z}-\tau_{z} \check{\Sigma}[\check{G}] \tau_{z}\right) \check{G}=\check{1},
$$

where the self-energy $\check{\Sigma}$ depends on the effective field $\check{\mathcal{G}}$. A closed equation for $\breve{\mathcal{G}}$ can be derived by integrating out the field $\eta$ in Eq. (83). This procedure leads to the equation

$$
\begin{gathered}
\mathcal{U}=\int \mathcal{D} \check{\mathcal{G}} e^{i S[\check{\mathcal{G}}] / \hbar}, \\
S[\check{\mathcal{G}}] / \hbar=-i \operatorname{Tr} \ln \left(\mathcal{L} \tau_{z}-\tau_{z} \check{\Sigma}[\check{\mathcal{G}}] \tau_{z}\right)+W[\check{\mathcal{G}}],
\end{gathered}
$$

where $\operatorname{Tr}$ denotes both the matrix trace in the KeldyshNambu space and the integration over the time variables. Noticing that the action is large, $S[\check{\mathcal{G}}] \sim \lambda_{\mathrm{ph}}^{-1}$, we evaluate the integral in Eq. (88) within the saddle-point approximation (cf. Ref. 38). The corresponding saddle-point equation is derived by varying the action with respect to $\breve{\mathcal{G}}$, which yields

$$
\left(\mathcal{L} \tau_{z}-\tau_{z} \check{\Sigma}[\check{\mathcal{G}}] \tau_{z}\right) \check{\mathcal{G}}=\lambda_{\mathrm{ph}} \check{1} .
$$

Comparing Eqs. (87) and (89), we obtain the relation $\check{\mathcal{G}}$ $=\lambda_{\mathrm{ph}} \breve{G}$. Written in the terms of $\check{G}$,

$$
\left(\mathcal{L} \tau_{z}-\tau_{z} \check{\Sigma}\left[\lambda_{\mathrm{ph}} \check{G}\right] \tau_{z}\right) \check{G}=\check{1},
$$

the saddle-point equation is the Dyson equation for the qubit Keldysh-Green function (86) in which the self-energy contains only an undressed vertex part and a free phonon Green function. Including the parameter $\lambda_{\mathrm{ph}}$ back into the kernel $\check{D}$, $\lambda_{\text {ph }} \check{D} \rightarrow \check{D}$, we arrive at the expression for the self-energy (85), with $\check{\mathcal{G}}$ being replaced by $\check{G}$, while $\check{D}$ is given by Eq. (82).

To proceed with the derivation of the kinetic equation, it is convenient to introduce a triangular form for the KeldyshGreen function by performing a transformation in Keldysh space

$$
\check{G} \rightarrow \check{L} \tau_{z} \check{G} \check{L}^{-1}=\left(\begin{array}{cc}
G^{R} & G^{K} \\
0 & G^{A}
\end{array}\right), \quad \check{L}=\frac{1}{\sqrt{2}}\left(\begin{array}{cc}
1 & -1 \\
1 & 1
\end{array}\right),
$$

where $G^{R(A)}=G^{11}-G^{12(21)}$ is the retarded (advanced) Green function and $G^{K}=G^{11}+G^{22}=G^{12}+G^{21}$ is the Keldysh component. Similar relations also hold for the self-energy. Then Eq. (90) takes the form

$$
\check{\mathcal{L}} \check{G}=\check{1}+\check{\check{L} \check{G} .}
$$

A kinetic equation is obtained by considering the difference between Eq. (92) and its Hermitian conjugate for the Keldysh component ${ }^{36}$

$$
\begin{aligned}
& i \hbar\left(\partial_{t}+\partial_{t^{\prime}}\right) G^{K}\left(t, t^{\prime}\right)-E_{a}^{*}\left[\sigma_{z}, G^{K}\left(t, t^{\prime}\right)\right] \\
& \quad=\left(\Sigma^{R} G^{K}-G^{K} \Sigma^{A}+\Sigma^{K} G^{A}-G^{R} \Sigma^{K}\right)\left(t, t^{\prime}\right) .
\end{aligned}
$$

The right-hand side of Eq. (93) describes the qubit decoherence as well as dynamic corrections due to the phonons. ${ }^{40} \mathrm{In}$ the absence of the coupling to phonons, the solution of Eq. (93) has the form

$$
G_{0}^{K}\left(t, t^{\prime}\right)=(-i / \hbar) e^{-i E_{a}^{*} \sigma_{z} t / \hbar} F e^{i E_{a}^{*} \sigma_{z} t^{\prime} / \hbar},
$$

where $F$ is a time-independent matrix determined by the initial state of the qubit. When a weak interaction with the phonons is switched on, an asymptotic solution to Eq. (93) can be written in the form

$$
G^{K}\left(t, t^{\prime}\right)=(-i / \hbar) e^{-i E_{a}^{*} \sigma_{z} t / \hbar}\left\{F\left[\left(t+t^{\prime}\right) / 2\right]+\widetilde{F}\left(t, t^{\prime}\right)\right\} e^{i E_{a}^{*} \sigma_{z} t^{\prime} / \hbar},
$$

where the matrix $F$ is, on the time scale $\hbar / E_{a}^{*}$, a slowly evolving function of the global time $\left(t+t^{\prime}\right) / 2$ (Markovian approximation), and $\widetilde{F}$ is a rapidly oscillating smallamplitude correction (see Appendix B). Equations for the matrix elements of $F$ are derived in Appendix B, Eqs. (B14) and (B15), and for the diagonal matrix elements they read

$$
\partial_{t} F_{1}=-\partial_{t} F_{2}=-\frac{\nu}{2}\left[(2 N+1)\left(F_{1}-F_{2}\right)+F_{1} F_{2}-1\right],
$$

while for the off-diagonal matrix element $F_{12}=F_{21}^{*}$ the equation has the form

$$
\begin{gathered}
\partial_{t} F_{12}=-\left[\frac{\nu}{2}\left(2 N+1-F_{z}\right)+2 i\left(\delta+\delta_{0} F_{z}\right)\right] F_{12}, \\
F_{z}(t)=\left(F_{1}-F_{2}\right) / 2,
\end{gathered}
$$

where $\nu$ is the phonon-induced transition rate between the qubit levels 


$$
\nu=(2 \pi / \hbar) \int \frac{d^{3} q}{(2 \pi)^{3}} V\left|\gamma_{\mathbf{q}}^{*}\right|^{2} \delta\left(2 E_{a}^{*}-\hbar \Omega_{q}\right) .
$$

$N=\left(e^{2 \beta E_{a-1}^{*}}-1\right)^{-1}$ is the phonon distribution function at a frequency equal to the qubit level spacing $\beta=1 / \mathrm{kT}$ and $\delta$ and $\delta_{0}$ are small dynamic corrections defined in Eq. (B16).

For equal times $t=t^{\prime}, G^{K}(t, t)$ is related to the qubit density matrix (39) as follows: $G_{\sigma \sigma^{\prime}}^{K}(t, t)=(-i / \hbar)\left[2 \rho_{\sigma \sigma^{\prime}}(t)\right.$ $\left.-\delta_{\sigma \sigma^{\prime}}\right]$, and therefore Eqs. (96) and (97) in fact give the kinetic equation for the qubit density matrix in the interaction picture $\widetilde{\rho}=e^{i E_{a}^{*} \sigma_{z} t / \hbar} \rho e^{-i E_{a}^{*} \sigma_{z} t / \hbar}, F_{\sigma}=2 \rho_{\sigma \sigma}-1, F_{12}=2 \widetilde{\rho}_{12}$. It is instructive to write Eq. (96) in terms of the qubit occupation numbers $n_{\sigma}=1-\rho_{\sigma \sigma}$,

$$
\partial_{t} n_{1}=-\partial_{t} n_{2}=-\nu\left[(N+1) n_{1}\left(1-n_{2}\right)-N n_{2}\left(1-n_{1}\right)\right] .
$$

The right-hand side of this equation has the standard form of the electron-phonon collision term, yielding the Fermi distribution for the equilibrium occupation numbers

$$
n_{1,2}^{0}=\frac{1}{e^{ \pm \beta E_{a}^{*}+1}} .
$$

This conclusion is consistent with the well known fact that the Fermi distribution of the Andreev levels gives correct magnitude for the equilibrium Josephson current. ${ }^{28,29}$ Furthermore, it follows from Eqs. (99) and (100) that $\operatorname{Tr} \rho(t)$ $=1$. Then the equations for the two independent components of the density matrix $\rho_{z}=\left(\rho_{11}-\rho_{22}\right) / 2$, and $\tilde{\rho}_{12}$, omitting the dynamic corrections, are given by

$$
\begin{gathered}
\partial_{t} \rho_{z}=-\nu\left[(2 N+1) \rho_{z}-\rho_{z}^{2}-1 / 4\right], \\
\partial_{t} \widetilde{\rho}_{12}=-\nu\left(N+1 / 2-\rho_{z}\right) \widetilde{\rho}_{12} .
\end{gathered}
$$

These nonlinear equations are drastically different from the linear Bloch-Redfield equation describing the decoherence of macroscopic superconducting qubits, ${ }^{22}$ and they have qualitatively different solutions, as illustrated in Fig. 6. The exact solutions for Eqs. (101) and (102) read

$$
\delta \rho_{z}(t)=\frac{\delta \rho_{z}(0) e^{-\Gamma t}}{1+\delta \rho_{z}(0) \sinh \left(\beta E_{a}^{*}\right)\left(1-e^{-\Gamma t}\right)}, \quad \Gamma=\frac{\nu}{\sinh \left(\beta E_{a}^{*}\right)},
$$

$$
\widetilde{\rho}_{12}(t)=\frac{\rho_{12}(0) e^{-\Gamma t / 2}}{1+\delta \rho_{z}(0) \sinh \left(\beta E_{a}^{*}\right)\left(1-e^{-\Gamma t}\right)},
$$

where $\delta \rho_{z}(t)=\rho_{z}^{0}-\rho_{z}(t)$ is the deviation from the equilibrium $\rho_{z}^{0}=(1 / 2) \tanh \left(\beta E_{a}^{*} / 2\right)$. The evolutions of the diagonal (relaxation) and off-diagonal (dephasing) parts of the density matrix are qualitatively similar. One may distinguish the linear regime $\delta \rho_{z}(0) \sinh \left(\beta E_{a}^{*}\right) \ll 1$, when the decoherence is determined by the exponential law

$$
\delta \rho_{z}(t)=\delta \rho_{z}(0) e^{-\Gamma t}, \quad \tilde{\rho}_{12}(t)=\rho_{12}(0) e^{-\Gamma t / 2} .
$$

However, the decoherence rate $\Gamma$ becomes exponentially small at temperature smaller than the qubit level spacing

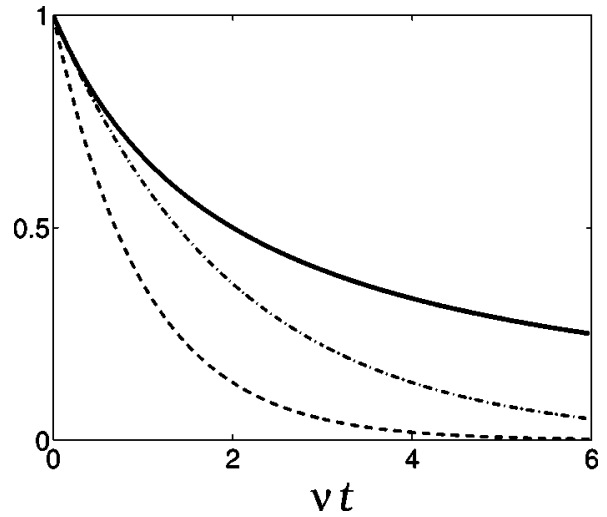

FIG. 6. Decay with time of a "cat" state $\left[\delta \rho_{z}(0)=\rho_{12}(0)=1 / 2\right]$. The bold line indicates the evolution of both (normalized) density matrix elements for ALQ for $1 / \beta=0.2 E_{a}^{*}$. For comparison, exponential relaxation and dephasing of a macroscopic superconducting qubit are illustrated with the dashed and dashed-dotted lines, respectively.

$\beta E_{a}^{*} \gg 1$. At this temperature, the most interesting is the opposite, nonlinear regime $\delta \rho_{z}(0) \sinh \left(\beta E_{a}^{*}\right) \gg 1$. In this case, there is a wide time interval $t \ll \sinh \left(\beta E_{a}^{*}\right) / \nu$, where both the relaxation and dephasing follow the power law (see Fig. 6)

$$
\delta \rho_{z}(t)=\frac{1}{\nu t}, \quad \widetilde{\rho}_{12}(t)=\frac{\rho_{12}(0)}{\delta \rho_{z}(0)} \frac{1}{\nu t},
$$

and only at very large times $t \gg \sinh \left(\beta E_{a}^{*}\right) / \nu$, the decoherence undergoes a crossover to an exponential regime similar to Eq. (105). We note that the exponentially small relaxation rate in the linear regime is well known for the quasiparticle recombination in bulk superconductors. ${ }^{41}$

\section{Evaluation of the transition rate}

We conclude our study with the evaluation of the phononinduced transition rate, $\nu$ in Eq. (98). To evaluate the transition rate, one needs to specify the geometry of the junction in greater detail. Let us suppose that our adiabatic constriction, Eq. (4), is formed by a hard-wall potential and has an axial symmetry. Under these assumptions, the Fourier component of the transverse wave function in Eq. (76) has the form

$$
F\left(\mathbf{q}_{\perp}, x\right)=2 \frac{J_{1}\left[r_{\perp}(x) q_{\perp}\right]}{r_{\perp}(x) q_{\perp}},
$$

where $r_{\perp}(x)$ is the radius of the constriction cross section. The magnitude of the relaxation rate essentially depends on the parameter $r_{\perp}(0) Q$, where $r_{\perp}(0)$ is the radius of the neck of the constriction and $Q=2 E_{a}^{*} / \hbar s$ is the wave vector of phonons responsible for the interlevel transitions; for atomicsize constrictions, this parameter is small, $r_{\perp}(0) Q \ll 1$. Let us assume that the qubit level spacing is not too small, $E_{a}^{*} / \Delta$ $\gg s / v$; then the phonon wave vector $Q$ is large compared to the inverse penetration length of the Andreev level wave function $Q \gg \kappa$.

Let us for a moment assume that the Andreev level wave function does not spread out in the electrodes, but remains 


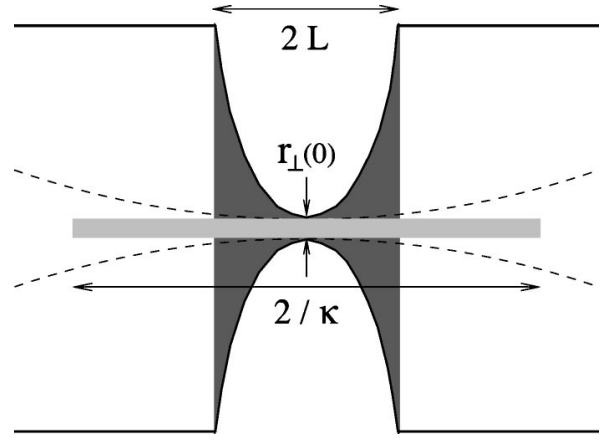

FIG. 7. Interaction region of the Andreev levels with phonons in short QPC (dark shadow) and long QPC (light shadow); in long QPC, increase of the constriction radius (dashed line) can be neglected.

confined in the transverse direction $r_{\perp}(x)=$ const $=r_{\perp}(0)$ (see Fig. 7); then the Fourier component in Eq. (107) is close to unity and the interaction region in Eq. (76) is limited by the penetration length of the Andreev state $x \sim 1 / \kappa$, restricting relevant phonon longitudinal wave vectors to small values $q_{x} \sim \kappa \ll Q$. The transition rate in this case reads

$$
\nu_{0}=\frac{\gamma^{2} R^{*}}{16 \hbar \rho s^{2}} \kappa Q^{2} \sim R *\left(\zeta_{e} / E_{a}^{*}\right) \tau_{\mathrm{ph}}^{-1}\left(E_{a}^{*}\right),
$$

where $\tau_{\mathrm{ph}}^{-1}\left(E_{a}^{*}\right) \sim E_{a}^{* 3} / \hbar \Theta_{D}^{2}$ is a bulk electron-phonon relaxation rate at the Andreev level energy $\left(\Theta_{D}\right.$ is the Debye temperature). This result has been derived in Ref. 32 (although neglecting the renormalization effect), and it can be qualitatively applied to long constrictions, whose length exceeds the coherence length. For the short constrictions considered here $L \ll 1 / \kappa$, the effect of spreading out of the Andreev level wave function is essential and the approximation $r_{\perp}=$ const is not applicable.

Let us adopt the following model for the constriction shape $r_{\perp}(x)=r_{\perp}(0)(1-|x| / L)^{-a}, a>1$. It is then easy to see that the integral in Eq. (76) will have a cut off at $|x|=L$ $\ll 1 / \kappa$. The function $J_{1}\left(z^{a}\right) / z^{a}$ in Eq. (107) can with good accuracy be approximated with the function $(1 / 2) \theta\left(z_{0}-z\right)$, where $z_{0} \approx 1$ and the integral in Eq. (76) is easily evaluated, giving

$$
\nu=\kappa L \nu_{0},
$$

i.e., the transition rate in short constrictions is significantly reduced. This is the effect of the small spatial region available for the Andreev level-phonon interaction in short QPC.

\section{CONCLUSION}

Let us summarize the outlined theory for the Andreev level qubit (ALQ). The ALQ belongs to the family of superconducting flux qubits, but it differs from the macroscopic flux qubits ${ }^{3-6}$ in several important respects. First, the quantum hybridization of the flux (and persistent current) states in the ALQ loop is produced by electronic backscattering in the quantum point contact (QPC) rather than by charge fluctua- tions on the junction capacitors in the case of the macroscopic qubits. Thus, in principle, neither small junction capacitance nor large loop inductance is critical for the ALQ operation. Secondly, the ALQ is based on a QPC with large, almost full, transparency, in contrast to classical tunnel junctions employed in the macroscopic superconducting qubits. Large contact transparency is required for placing Andreev levels deep within the superconducting energy gap to achieve good decoupling from the continuum electronic states. To guarantee good separation of the qubit levels from the continuum, the amplitude of the phase fluctuations around the biasing point $\phi \approx \pi$ must be restricted to small values, which implies small inductance of the qubit loop.

In the tunnel junctions of the macroscopic qubits, Andreev levels are fast variables whose effect, after averaging, reduces to the Josephson potential energy added to the Hamiltonian of the loop oscillator. In the transparent junction of the ALQ, Andreev levels are slow variables which cannot be averaged out and the full description includes the Andreev two-level Hamiltonian strongly coupled to the quantum loop oscillator. Derivation of the effective two-level Hamiltonian goes beyond the tunnel model approximation and is done by incorporating the exact boundary condition into the action of the contact.

The qubit read out is performed by measuring (fluctuating) persistent current or induced flux in the qubit loop. To simplify the interpretation of the measurements, the loop plasma frequency is supposed to be large compared to the qubit frequency so that the loop oscillator is "enslaved" by the Andreev levels making the current to directly follow the Andreev level evolution. Other regimes, e.g., resonance between the Andreev levels and the loop oscillator, can also be considered but they remain outside the scope of this paper.

Typical circuit parameters for the ALQ could be chosen as follows: The Josephson coupling energy for a single open conducting mode is quite large $E_{J} \approx \Delta$, giving considerable Josephson critical current $I_{c} \sim 400 \mathrm{nA}$ for $\mathrm{Nb}$. For a bare contact reflectivity $R \leqslant 0.01$ and a contact capacitance $C$ $\sim 0.1 \mathrm{pF}$, and a loop inductance $L \sim 0.1 \mathrm{nH}$, the loop plasma frequency $\omega_{p} \sim 10^{11} \mathrm{~s}^{-1}$ and the inductive energy $E_{L} / \hbar$ $\sim 10^{13} \mathrm{~s}^{-1} \sim 10 \Delta_{N b} / \hbar$, exceed, as required, the qubit level spacing $E_{a}^{*} / \hbar \sim 10^{10} \mathrm{~s}^{-1}$. In temperature units, the latter corresponds to approximately $100 \mathrm{mK}$. Therefore, at typical experimental temperatures of $10-20 \mathrm{mK}$ the system should exhibit pronounced quantum dynamics.

In the absence of microscopic interaction of the Andreev levels with other microscopic modes in the junction, a full description of the ALQ dynamics is given by a reduced single-particle electronic density matrix. This concerns the qubit manipulation with external fields, qubit measurement, and qubit-qubit coupling. It is important to emphasize that the fermionic origin of the Andreev levels does not affect this macroscopic behavior of the system, which is adequately described with the two-level density matrix and the Liouville equation similar to any other qubit.

Even if good decoupling of the qubit states from the continuum electronic states is achieved, there are still soft microscopic modes in the junction that could couple to the Andreev levels. These modes present a potential source of "intrinsic" decoherence, in addition to the commonly consid- 
ered external decoherence, e.g., due to fluctuating biasing and read out circuits. We have considered such intrinsic decoherence of the ALQ related to acoustic phonons under the simplest assumptions about the phonon equilibrium and the Markovian evolution. It turns out that the collision terms in the kinetic equation are nonlinear, in contrast to the linear master equation for the macroscopic superconducting qubits. $^{22}$ This reflects the fermionic nature of the Andreev states and leads to considerable enhancement of the decoherence time at low temperature. One can understand this effect in the following way. Andreev levels belong to a many-body system of superconducting electrons. Although the macroscopic behavior of the ALQ can be expressed in terms of the single-particle density matrix, the microscopic interaction with phonons involves two-particle correlation functions, which are sensitive to the fermionic nature of the Andreev states and obey the Pauli exclusion principle. This leads to a reduced probability of phonon-induced interlevel transitions and hence to a slower decoherence.

Furthermore, the rate of phonon-induced transitions between the Andreev levels is significantly reduced compared to the bulk transition rate. The reason is that both the Andreev levels belong to the same normal electronic mode; this together with a rapid spreading out of the Andreev level wave function in the contact electrode strongly reduces the relevant phonon phase space.

In this paper, only the case of a single-mode QPC was considered for clarity. However, the approach might also be relevant for macroscopic Josephson qubits with tunnel junctions. In junctions with disordered tunnel barriers, open conducting modes with large transmissivity are present. ${ }^{42,43}$ This introduces low-energy Andreev levels, which implies that quantum phase fluctuations become coupled to these Andreev levels, and the system must be described with the effective ALQ-type Hamiltonian. Finally, the effective ALQ Hamiltonian might be appropriate for $d$-wave qubits for geometries where low-energy Andreev levels, midgap states, ${ }^{44}$ build up in the junction.

\section{ACKNOWLEDGMENT}

We acknowledge useful discussions at various stages of our work with D. Khmelnitsky, Yu. Galperin, G. Johansson, A. Zaikin, L. Gorelik, R. Shekhter, I. Krive, and E. Bezuglyi. Support from EU-SQUBIT and SQUBIT-2 consortia, the Swedish Science Foundation, SSF-OXIDE consortium, and KVA is gratefully acknowledged.

\section{APPENDIX A: TUNNEL LIMIT FOR QPC}

In this appendix, we consider a low transparency QPC $D \ll 1$ and apply our method to recover the results of Refs. 18 and 19 for tunnel Josephson junctions. In this limit, the Andreev levels lie very close to the edges of the superconducting gap $E_{a} \approx \Delta$, and therefore the field $\eta$ is a fast variable, which has to be integrated out along with all the other electronic degrees of freedom. This will result in an effective action for the phase difference alone. After integration over the electronic fields, the propagator in Eq. (22) takes the form

$$
\begin{gathered}
\mathcal{U}_{e}[\phi]=\int \mathcal{D}^{2} \eta \mathcal{D}^{2} \psi_{L, R} e^{i S_{e} / \hbar}=\exp \{i S[\phi] / \hbar\}, \\
S[\phi] / \hbar=-i \operatorname{Tr} \ln \left(1+\frac{D}{(1+\sqrt{R})^{2}} g^{-1} e^{-i \sigma_{z} \phi / 2} g e^{i \sigma_{z} \phi / 2}\right) .
\end{gathered}
$$

Here $g$ is the Green function defined in Eq. (32),

$$
g(t)=-(1 / \hbar \mathrm{v}) \int_{-\infty}^{+\infty} \frac{d \omega}{2 \pi} e^{-i \omega t} \frac{\hbar \omega+\Delta \sigma_{x}}{\sqrt{\Delta^{2}-\hbar^{2}(\omega+i \operatorname{sgn} \omega 0)^{2}}},
$$

and the matrix product in Eq. (A2) also includes the time convolutions. Taking advantage of the small $D$, and expanding the action (A2), the lowest order term reads

$$
\begin{aligned}
S[\phi] / \hbar= & -i(D / 4) \operatorname{tr} \int_{-\infty}^{+\infty} d t_{1} d t_{2} g^{-1}\left(t_{1}-t_{2}\right) e^{-i \sigma_{z} \phi\left(t_{2}\right) / 2} g\left(t_{2}\right. \\
& \left.-t_{1}\right) e^{i \sigma_{z} \phi\left(t_{1}\right) / 2},
\end{aligned}
$$

where the trace refers to the Nambu space. After taking the trace, the action can be written in the following form:

$$
\begin{aligned}
S[\phi] / \hbar= & \int_{-\infty}^{+\infty} d t_{1} d t_{2}\left[\alpha\left(t_{1}-t_{2}\right) \cos \frac{\phi\left(t_{1}\right)-\phi\left(t_{2}\right)}{2}\right. \\
& \left.+\beta\left(t_{1}-t_{2}\right) \cos \frac{\phi\left(t_{1}\right)+\phi\left(t_{2}\right)}{2}\right],
\end{aligned}
$$

with the kernels $\alpha$ and $\beta$ given by

$$
\begin{gathered}
\alpha(t)=i(D / 2)(\Delta / 2 \hbar)^{2}\left[H_{1}^{(1)}(t \Delta / \hbar)\right]^{2}, \\
\beta(t)=-i(D / 2)(\Delta / 2 \hbar)^{2}\left[H_{0}^{(1)}(t \Delta / \hbar)\right]^{2},
\end{gathered}
$$

where $H_{0,1}^{(1)}$ are Hankel functions of the first kind. Analytical continuation to imaginary time in Eqs. (A6) and (A7) using the relation $K_{\nu}(t)=(\pi / 2) i^{1+\nu} H_{\nu}^{(1)}(i t)$ leads to the same expressions for the kernels $\alpha$ and $\beta$ as derived in Refs. $18,19,34$, for tunnel Josephson junctions [with normal resistance of the tunnel junction being replaced by the normal resistance of the single-channel QPC: $\left.R_{N}=\left(D e^{2} / \pi \hbar\right)^{-1}\right]$.

For a slowly varying phase, $\phi$, on the time scale of the kernel variations $\hbar / \Delta$ both cosine terms in Eq. (A5) can be expanded with respect to the relative time coordinate $\tau=t_{1}$ $-t_{2}$ up to the second order, and the effective action then takes a simpler form

$$
S[\phi]=\int d t\left[\frac{\delta C}{2}\left(\frac{\hbar \dot{\phi}}{2 e}\right)^{2}+\frac{\hbar}{2 e} I_{c} \cos \phi\right],
$$

where $I_{c}=2 e \int_{-\infty}^{+\infty} d \tau \beta(\tau)=e D \Delta / 2 \hbar$ is the critical Josephson current of the single-channel tunnel point contact and 


$$
\begin{aligned}
\delta C(\phi) & =-\frac{e^{2}}{\hbar} \int_{-\infty}^{+\infty} d \tau \tau^{2}[\alpha(\tau)-\beta(\tau) \cos \phi] \\
& =\frac{3}{32} \frac{D e^{2}}{\Delta}[1-(1 / 3) \cos \phi]
\end{aligned}
$$

is the correction to the contact capacitance due to quasiparticle tunneling. ${ }^{34,19}$

\section{APPENDIX B: DERIVATION OF THE KINETIC EQUATION}

In this Appendix we derive Eqs. (96) and (97) from the equation for the Keldysh function (93). It is convenient to write the equation in the mixed representation

$$
\begin{aligned}
& i \hbar \partial_{t} G_{\omega}^{K}(t)-E_{a}^{*}\left[\sigma_{z}, G_{\omega}^{K}(t)\right] \\
& \quad=\left(\Sigma^{R} G^{K}-G^{K} \Sigma^{A}+\Sigma^{K} G^{A}-G^{R} \Sigma^{K}\right)_{\omega}(t),
\end{aligned}
$$

where

$$
\check{G}_{\omega}(t)=\int d \tau e^{i \omega \tau} \check{G}(t+\tau / 2, t-\tau / 2)
$$

and the products include the convolutions defined by the equation

$$
\begin{aligned}
(A B)_{\omega}(t)= & \int \frac{d \omega_{1} d \omega_{2}}{(2 \pi)^{2}} \int d \tau_{1} d \tau_{2} e^{i\left[\left(\omega-\omega_{1}\right) \tau_{2}+\left(\omega-\omega_{2}\right) \tau_{1}\right]} A_{\omega_{1}}(t \\
& \left.+\tau_{1} / 2\right) B_{\omega_{2}}\left(t-\tau_{2} / 2\right)
\end{aligned}
$$

Neglecting the effect of the phonons, we replace the retarded and advanced Green functions of the qubit $G^{R, A}$ in the righthand side of Eq. (B1) by the free Green functions $G_{\omega}^{R, A}$ $=\left(\hbar \omega-E_{a}^{*} \sigma_{z} \pm i 0\right)^{-1}$, which are time independent in the mixed representation. Thus, the time dependence in the self-energy only comes from the Keldysh component $G^{K}$. The selfenergy components take the form

$$
\begin{aligned}
\sum_{\omega}^{R(A)}(t) & =(i \hbar / 2) \sigma_{x} \int \frac{d \Omega}{2 \pi}\left[D_{\Omega}^{K} G_{\omega-\Omega}^{R(A)}+D_{\Omega}^{R(A)} G_{\omega-\Omega}^{K}(t)\right] \sigma_{x}, \\
\sum_{\omega}^{K}(t) & =(i \hbar / 2) \sigma_{x} \int \frac{d \Omega}{2 \pi}\left(D_{\Omega}^{R}-D_{\Omega}^{A}\right)\left[\left(2 N_{\Omega}+1\right) G_{\omega-\Omega}^{K}(t)\right. \\
& \left.+G_{\omega-\Omega}^{R}-G_{\omega-\Omega}^{A}\right] \sigma_{x},
\end{aligned}
$$

where

$$
D_{\Omega}^{R, A}=\int \frac{d \Omega^{\prime}}{2 \pi \hbar} \frac{\mathcal{D}_{\Omega^{\prime}}}{\Omega-\Omega^{\prime} \pm i 0}, \quad D_{\Omega}^{K}=-(i / \hbar)\left(2 N_{\Omega}+1\right) \mathcal{D}_{\Omega},
$$

$N_{\Omega}=\left(e^{\beta \hbar \Omega}-1\right)^{-1}$ is the equilibrium phonon distribution function, and $\mathcal{D}_{\Omega}$ is the spectral weight function of the phonon bath

$$
\mathcal{D}_{\Omega}=2 \pi \operatorname{sgn}(\Omega) \sum_{\mathbf{q}}\left|\gamma_{\mathbf{q}}^{*}\right|^{2} \delta\left(|\Omega|-\Omega_{q}\right)
$$

After integrating Eq. (B1) over $\omega$, we obtain equation for the Keldysh function at coinciding times

$$
G^{K}(t)=\int \frac{d \omega}{2 \pi} G_{\omega}^{K}(t)
$$

$$
i \hbar \partial_{t} G^{K}(t)-E_{a}^{*}\left[\sigma_{z}, G^{K}(t)\right]=I_{0}+I_{1}(t)+I_{2}(t),
$$

where $I_{0}=\left(1 / 2 \hbar^{2}\right) \mathcal{D}_{2 E_{a}^{*} / \hbar} \sigma_{z}$ and

$$
\begin{aligned}
I_{1}(t)= & \frac{-i}{2 \hbar} \frac{d \Omega d \omega}{(2 \pi)^{2}} \int_{0}^{+\infty} d \tau\left(2 N_{\Omega}+1\right) \mathcal{D}_{\Omega}\left[e^{i\left(\omega-\Omega+E_{a}^{*} \sigma_{z} / \hbar\right) \tau} G_{\omega}^{K}(t\right. \\
& \left.-\tau / 2)-e^{i\left(\omega+\Omega-E_{a}^{*} \sigma_{z} / \hbar\right) \tau} \sigma_{x} G_{\omega}^{K}(t-\tau / 2) \sigma_{x}-\text { H.c. }\right],
\end{aligned}
$$

$$
\begin{aligned}
I_{2}(t)= & \int \frac{d \Omega d \omega_{1} d \omega_{2}}{2(2 \pi)^{3}} \int_{0}^{+\infty} d \tau \mathcal{D}_{\Omega}\left[e^{i\left(\omega_{2}-\omega_{1}-\Omega\right) \tau} \sigma_{x} G_{\omega_{1}}^{K}(t\right. \\
& \left.-\tau / 2) \sigma_{x} G_{\omega_{2}}^{K}(t-\tau / 2)+\text { H.c. }\right] .
\end{aligned}
$$

For weak electron-phonon interaction, the right-hand side of Eq. (B9) is a small perturbation, which allows one to construct an asymptotic solution by using an improved perturbation expansion

$$
\begin{aligned}
G_{\omega}^{K}(t)= & -(2 \pi i / \hbar) e^{-i E_{a}^{*} \sigma_{z} t / \hbar}\left(F_{\omega}(\lambda t)+\sum_{n=1}^{\infty} \lambda^{n} \widetilde{F}_{\omega}^{(n)}(t)\right) e^{i E_{a}^{*} \sigma_{z} t / \hbar}, \\
F_{\omega}(t)= & \delta\left(\omega-E_{a}^{*} \sigma_{z} / \hbar\right)\left(\begin{array}{cc}
F_{1}(t) & 0 \\
0 & F_{2}(t)
\end{array}\right)+\delta(\omega) \\
& \times\left(\begin{array}{cc}
0 & F_{12}(t) \\
F_{12}^{*}(t) & 0
\end{array}\right) .
\end{aligned}
$$

In Eq. (B12), $\lambda$ is a formal perturbation parameter that reflects weak electron-phonon interaction $\sim \mathcal{D}_{\Omega}$ and allows one to develop a systematic perturbative expansion. In the zeroorder approximation with respect to $\lambda$, the (timeindependent) matrix $F$ in Eq. (B13) is the solution of Eq. (B9) without the right-hand side. The first-order equation determines the time dependence in this matrix (which absorbs terms that formally diverge with time in a straightforward perturbative expansion),

$$
\partial_{t} F_{1}=-\partial_{t} F_{2}=-\frac{\nu}{2}\left[(2 N+1)\left(F_{1}-F_{2}\right)+F_{1} F_{2}-1\right],
$$

$$
\partial_{t} F_{12}=-\left[\frac{\nu}{2}\left(2 N+1-F_{z}\right)+2 i\left(\delta+\delta_{0} F_{z}\right)\right] F_{12},
$$




$$
F_{z}(t)=\left(F_{1}-F_{2}\right) / 2,
$$

where $\nu=\mathcal{D}_{2 E_{a}^{*} / \hbar} / \hbar^{2}$ is the phonon-induced transition rate between the qubit levels $N=N_{2 E_{a}^{*} / \hbar}$ and the quantities

$$
\begin{gathered}
\delta=\left(1 / 2 \hbar^{2}\right) f \frac{d \Omega}{d \pi}\left(2 N_{\Omega}+1\right) \frac{D_{\Omega}}{\Omega+2 E_{a}^{*} / \hbar}, \\
\delta_{0}=\left(1 / 2 \hbar^{2}\right) f \frac{d \Omega}{2 \pi} \frac{D_{\Omega}}{\Omega+2 E_{a}^{*} / \hbar}
\end{gathered}
$$

determine the phonon-induced shift of the qubit frequency. The higher order equations determine the rapidly oscillating terms $\widetilde{F}_{\omega}^{(n)}$ in Eq. (B12); e.g., the equation for $\widetilde{F}_{\omega}^{(1)}$ reads

$$
\partial_{t} \widetilde{F}^{(1)}=\mathcal{I}_{z} \sigma_{z}+\mathcal{I}_{+} \sigma_{+}+\mathcal{I}_{-} \sigma_{-},
$$

where $\sigma_{ \pm}=(1 / 2)\left(\sigma_{x} \pm i \sigma_{y}\right)$

$$
\begin{gathered}
\mathcal{I}_{z}=e^{-4 i E_{a}^{*} t / \hbar}\left(\nu / 4-i \delta_{0}\right) F_{12}^{2}(t)+\text { c.c. } \\
\mathcal{I}_{-}=\mathcal{I}_{+}^{*}=e^{-4 i E_{a}^{*} t / \hbar}\left[\nu(N+1 / 2)+2 i\left(\delta+\widetilde{\delta}_{0} F_{z}\right)\right] F_{12}(t), \\
\widetilde{\delta}_{0}=(1 / 2 \hbar)^{2} \int \frac{d \Omega}{2 \pi} \frac{D_{\Omega}}{\Omega} .
\end{gathered}
$$

It follows from these equations that $\widetilde{F}^{(1)}$ indeed rapidly oscillates, with the frequency $4 E_{a}^{*} / \hbar$ and has relatively small amplitude, proportional to $\hbar \nu / E_{a}^{*}$.
${ }^{1}$ J. Lantz, V. S. Shumeiko, E. N. Bratus, and G. Wendin, Physica C 368, 315 (2002).

${ }^{2}$ A. Zazunov, V. S. Shumeiko, E. N. Bratus', J. Lantz, and G. Wendin, Phys. Rev. Lett. 90, 087003 (2003).

${ }^{3}$ C. H. van der Wal, A. C. J. ter Haar, F. K. Wilhelm, R. N. Schouten, C. J. P. M. Harmans, T. P. Orlando, Seth Lloyd, and J. E. Mooij, Science 290, 773 (2000).

${ }^{4}$ J. R. Friedman, V. P. W. Chen, S. K. Tolpygo, and J. E. Lukens, Nature (London) 406, 43 (2000).

${ }^{5}$ I. Chiorescu, Y. Nakamura, C. J. P. M. Harmans, and J. E. Mooij, Science 299, 1869 (2003).

${ }^{6}$ D. Vion, A. Aassime, A. Cottet, P. Joyez, H. Pothier, C. Urbina, D. Esteve, and M. H. Devoret, Science 296, 886 (2002).

${ }^{7}$ V. S. Shumeiko, G. Wendin, and E. N. Bratus', Phys. Rev. B 48, 13129 (1993).

${ }^{8}$ V. S. Shumeiko, E. N. Bratus', and G. Wendin, in Proceedings of the XXXVI Moriond Conference, Les Arcs, 2001, edited by T. Martin, G. Montambaux, and J. Tran Thanh Van (EDP Sciences, Les Ulis, France, 2001), p. 521.

${ }^{9}$ B. E. Kane, Nature (London) 393, 133 (1998).

${ }^{10}$ D. Loss and D. P. DiVincenzo, Phys. Rev. A 57, 120 (1998).

${ }^{11}$ N. van der Post, E. T. Peters, I. K. Yanson, and J. M. van Ruitenbeek, Phys. Rev. Lett. 73, 2611 (1994).

${ }^{12}$ B. J. Vleeming, C. J. Muller, M. C. Koops, and R. de Bruyn Ouboter, Phys. Rev. B 50, 16741 (1994).

${ }^{13}$ H. Takayanagi, T. Akazaki, and J. Nitta, Phys. Rev. Lett. 75, 3533 (1995).

${ }^{14}$ E. Scheer, P. Joyez, D. Esteve, C. Urbina, and M. H. Devoret, Phys. Rev. Lett. 78, 3535 (1997).

${ }^{15}$ B. Ludoph, N. van der Post, E. N. Bratus', E. V. Bezuglyi, V. S. Shumeiko, G. Wendin, and J. M. van Ruitenbeek, Phys. Rev. B 61, 8561 (2000).

${ }^{16}$ M. F. Goffman, R. Cron, A. Levy Yeyati, P. Joyez, M. H. Devoret, D. Esteve, and C. Urbina, Phys. Rev. Lett. 85, 170 (2000).

${ }^{17}$ M. C. Koops, G. V. van Duyneveldt, and R. de Bruyn Ouboter, Phys. Rev. Lett. 77, 2542 (1996).

${ }^{18}$ V. Ambegaokar, U. Eckern, and G. Schön, Phys. Rev. Lett. 48, 1745 (1982).
${ }^{19}$ U. Eckern, G. Schön, and V. Ambegaokar, Phys. Rev. B 30, 6419 (1984).

${ }^{20}$ G. Schön and A. D. Zaikin, Phys. Rep. 198, 237 (1990).

${ }^{21}$ C. P. Slichter, Principles of Magnetic Resonance (SpringerVerlag, New York, 1990).

${ }^{22}$ Yu. Makhlin, G. Schön, and A. Shnirman, Rev. Mod. Phys. 73, 357 (2001).

${ }^{23}$ V. S. Shumeiko, E. N. Bratus', and G. Wendin, Low Temp. Phys. 23, 249 (1997).

${ }^{24}$ V. P. Galaiko, J. Low Temp. Phys. 26, 483 (1977).

${ }^{25}$ S. A. Artemenko and A. F. Volkov, Sov. Phys. Usp. 22, 295 (1979).

${ }^{26}$ A. G. Aronov, Yu. M. Galperin, V. L. Gurevich, and V. I. Kozub, in Nonequilibrium Superconductivity, edited by D. N. Langenberg and A. I. Larkin (North-Holland, Amsterdam, 1986), p. 325 .

${ }^{27}$ I. O. Kulik and A. N. Omel'yanchuk, Sov. J. Low Temp. Phys. 3, 459 (1977); 4, 142 (1978).

${ }^{28}$ A. Furusaki and M. Tsukada, Physica B 165-166, 967 (1990).

${ }^{29}$ C. W. J. Beenakker, and H. van Houten, Phys. Rev. Lett. 66, 3056 (1991).

${ }^{30}$ D. A. Ivanov and M. V. Feigelman, Phys. Rev. B 59, 8444 (1999).

${ }^{31}$ D. V. Averin, Phys. Rev. Lett. 82, 3685 (1999).

${ }^{32}$ D. A. Ivanov and M. V. Feigelman, JETP Lett. 68, 890 (1998).

${ }^{33}$ M. A. Desposito and A. L. Yeyati, Phys. Rev. B 64, 140511(R) (2001).

${ }^{34}$ A. I. Larkin and Yu. N. Ovchinnikov, Phys. Rev. B 28, 6281 (1983).

${ }^{35}$ A. Martin-Rodero, A. Levy Yeyati, and F. J. Garcia-Vidal, Phys. Rev. B 53, R8891 (1996).

${ }^{36}$ E. M. Lifshits and L. P. Pitaevsky, Physical Kinetics (Pergamon, Oxford, 1981).

${ }^{37}$ L. V. Keldysh, Sov. Phys. JETP 20, 1018 (1965).

${ }^{38}$ A. Kamenev and A. Andreev, Phys. Rev. B 60, 2218 (1999).

${ }^{39}$ Y. Avishai, A. Golub, and A. D. Zaikin, Phys. Rev. B 63, 134515 (2001).

${ }^{40}$ J. Rammer and H. Smith, Rev. Mod. Phys. 58, 323 (1986). 
${ }^{41}$ S. B. Kaplan, C. C. Chi, D. N. Langenberg, J. J. Chang, S. Jafarey, and D. J. Scalapino, Phys. Rev. B 14, 4854 (1976).

${ }^{42}$ K. M. Schep and G. E. W. Bauer, Phys. Rev. Lett. 78, 3015 (1997).
${ }^{43}$ Y. Naveh, V. Patel, D. V. Averin, K. K. Likharev, and J. E. Lukens, Phys. Rev. Lett. 85, 5404 (2000).

${ }^{44}$ T. Löfwander, V. Shumeiko, and G. Wendin, Supercond. Sci. Technol. 14, R53 (2001). 